\title{
Distinguishing disks from mergers: Tracing the kinematic asymmetries in local (U)LIRGs using kinemetry-based criteria
}

\author{
Enrica Bellocchi ${ }^{1,2}$, Santiago Arribas ${ }^{2}$, and Luis Colina ${ }^{2}$ \\ 1 Departamento de Física Teórica, Universidad Autónoma de Madrid, 28049 Madrid, Spain
2 Centro de Astrobiología, Departamento de Astrofísica, CSIC-INTA, Cra. de Ajalvir km.4, 28850 Torrejón de Ardoz, Madrid, Spain
e-mail: bellochie@cab.inta-csic. es; enrica. bellocchi@gmail. com
}

Received 16 July 2015 / Accepted 26 February 2016

\begin{abstract}
Context. The kinematic characterization of different galaxy populations is a key observational input for distinguishing between different galaxy evolutionary scenarios because it helps to determine the number ratio of rotating disks to mergers at different cosmic epochs. Local (ultra) luminous infrared galaxies ((U)LIRGs) cover similar range of star formation rates (SFR) as normal high redshift (high-z), star-forming galaxies (SFGs). Therefore, their study offer a unique opportunity to study at high linear resolution and signal-to-noise $(\mathrm{S} / \mathrm{N})$ extreme star forming events and compare these events with those observed at high-z.

Aims. Our goal is to analyze in detail the kinematics of the ionized gas as traced by the H $\alpha$ emission of a large sample of 38 local $(z<0.1)$ (U)LIRG systems (50 individual galaxies). In this study, we apply kinematic criteria, which are able to characterize the evolutionary status of these systems, allowing us to derive the disk and merger ratio in such local systems.

Methods. We obtained Very Large Telescope (VLT) VIMOS optical integral field spectroscopy (IFS) data of a sample of 38 (U)LIRGs These systems are morphologically classified in four groups according to their dynamical phases: isolated disk, paired disk, ongoing merger, and post-coalescence merger. The first two are referred as "disk", while the second two are referred to as "merger". The "unweighted" and "weighted" kinemetry-based methods are used to kinematically classify our galaxies in disk and merger. The total kinematic asymmetry value $K_{\text {tot }}$ has been used to quantify the global kinematic asymmetry degree of the observed and simulated systems.

Results. From the kinemetry-based analysis we are able classify our local (U)LIRGs in three distinct kinematic groups according to their total kinematic asymmetry values $\left(K_{\text {tot }}\right)$ as derived when using the weighted (unweighted) method: (1) 25 out of 50 galaxies are kinematically classified as disk with a $K_{\text {tot }} \leq 0.16(0.14)$; (2) 9 out of 50 galaxies are kinematically classified as merger with a $K_{\text {tot }} \geq 0.94$ (0.66); (3) 16 out of 50 galaxies lie in the "transition region", in which disks and mergers coexist, with $0.16(0.14)<$ $K_{\text {tot }}<0.94(0.66)$. The $K_{\text {tot }}$ frontier value that better classifies the highest numbers of disks and mergers, in agreement with their morphology, is $K_{\text {tot }}=0.19(\sim 0.15)$ : using this value the fractions of "well-classified disks" $\left(I_{\text {disk }}\right)$ vs. "well classified mergers" $\left(I_{\text {merger }}\right)$ are, respectively, $\sim 80 \%$ vs. $\sim 100 \%$. The same results are obtained if we only consider the isolated disks as "true disks". When we apply our criteria to the high- $z$ simulated systems, a lower total kinematic asymmetry frontier value $\left(K_{\text {tot }} \sim 0.16(\sim 0.14)\right)$ is derived with respect to that found locally. The loss of angular resolution smears out the kinematic features, thus making objects to appear more kinematically regular (disky) than actually they are.
\end{abstract}

Key words. Galaxy: evolution - Galaxy: kinematics and dynamics

\section{Introduction}

In the standard model of hierarchical galaxy assembly, mergers are the dominant source of mass accretion and growth in massive high-redshift (high-z) galaxies (Cole et al. 2000; Somerville et al. 2001). In this scenario, galaxies are assumed to form at the center of dark matter halos as the baryonic gas cools (e.g., Baugh 2006), and their subsequent evolution is controlled by the merging histories of the halos containing them (e.g., Cole et al. 1994). As derived from the observations, major merging is undoubtedly taking place at high- $z$ (e.g., Tacconi et al. 2006, 2008). More recently, Kartaltepe et al. (2012), studying a sample of ULIRGs at $z \sim 2$, found that the majority of the sources show signs of major mergers. These mechanisms support the hypothesis that gasrich late-type galaxies can transform into gas-poor early-type E/S0 galaxies, as predicted using detailed simulations (Mihos \& Hernquist 1996; Combes 2004; Conselice 2006). As a result of this framework, we expect to find galaxies characterized by complex and disturbed kinematics, such as distorted and asymmetric velocity fields, as a proof of a strong ongoing interaction.
On the other hand, hydrodynamical simulations (Robertson et al. 2006; Governato et al. 2007, 2009; Hopkins et al. 2009a,b) have shown that the gas fraction at fusion time and the amount of dissipation in a major merger of disk galaxies is a key parameter to generate a bulge dominated/elliptical (i.e., through a dry merger) or a spiral galaxy (i.e., through a gas-dominated merger). Indeed, according to this scenario, a disk can be reformed in the remnant when the fraction of gas at the fusion time is higher than 50\% (e.g., Hammer et al. 2009; Puech et al. 2012).

In the last few years, many works found that most of the high- $z$ galaxies are revealed as disk-like, rotating velocity field patterns, although they appear to be turbulent (i.e., Lehnert et al. 2009; Burkert et al. 2010) as given by their high velocity dispersion (i.e., $\sigma=30-100 \mathrm{~km} \mathrm{~s}^{-1}$ ) and low dynamical ratio $(v / \sigma<1$; Genzel et al. 2008; Förster Schreiber et al. 2009; Wisnioski et al. 2011). In order to explain their kinematic patterns in new models of disk formation at high- $z$, recent theoretical works (i.e., Kereš et al. 2005; Davé 2008; Genel et al. 2008; Dekel et al. 2009a; Ceverino et al. 2010), based 
on semianalytical approaches and hydrodynamical simulations, have invoked a rapid, but more continuous gas accretion via cold flows and/or minor mergers that are able to supply gas directly to the center of the galaxies (i.e., Kereš et al. 2005; Ocvirk et al. 2008; Dekel et al. 2009a). These cold flows and minor mergers likely play an important role in driving the mass growth of a massive star-forming galaxy (SFG) at high-z (e.g., Daddi et al. 2007; Elbaz et al. 2007; Noeske et al. 2007). One of the first pieces of evidence of the "clumpy disk" picture came from the work of Förster Schreiber et al. (2006), who observed the $\mathrm{H} \alpha$ emission of a sample of $14 \mathrm{BM} / \mathrm{BX}$ galaxies. These authors confirmed the presence of a significant fraction of galaxies with rotation fields characteristic of disks large enough to be resolved in 0.5 arcsec seeing. A large portion of the strongly SFGs at $z=1-3$ do not show the disturbed kinematics expected according to the hierarchical model but these galaxies are characterized by regularly rotating disks (e.g., Cresci et al. 2009; Epinat et al. 2009; Förster Schreiber et al. 2009; Gnerucci et al. 2011). This result has suggested that even stronger star formation may be fueled by the accretion of pristine gas from the halo and by dynamical instabilities within the massive gaseous disks (Genel et al. 2008; Dekel et al. 2009b; Bouché et al. 2010; Cresci et al. 2010).

The discrepancies between morphological and kinematical results have increased the importance of kinematic studies because objects that are photometrically irregular in broadband HST images show "regular" kinematic maps (Bournaud et al. 2008; van Starkenburg et al. 2008; Puech 2010; Jones et al. 2010; Förster Schreiber et al. 2011; Genzel et al. 2011). Thus, the aforementioned results emphasize the crucial role of spatially and spectrally resolved investigations of galaxies at different redshifts, such as those based on integral field spectroscopy (IFS), to map their morphology and kinematics.

A useful way to figure out which dominant scenario drives the galaxy evolution at different cosmic epochs is to estimate the number ratio of (rotating) disks to mergers (i.e., disk/merger). When applying different techniques, some discrepancies can raise in classifying several kinds of galaxies in disks and mergers. The first and most widely used technique was the visual morphological classification (e.g., Dasyra et al. 2008; Kartaltepe et al. 2010; Zamojski et al. 2011); then other classification methods, such as the estimate of asymmetry and clumpiness parameters (e.g., Conselice et al. 2003) and the use of the Gini-M 20 plane (e.g., Lotz et al. 2008), have been considered as well. In the last decade, measures of galaxy kinematics (e.g., Genzel et al. 2008; Förster Schreiber et al. 2006, 2009) have increased their importance. A visual (kinematic) classification was applied at intermediate redshift (i.e., $0.4 \leq z \leq 0.7$ ) by Flores et al. (2006) and Yang et al. (2008) to classify the systems kinematically; this classification was also applied locally by Bellocchi et al. (2013) to investigate the properties of velocity fields of galaxies observed with IFS.

The kinematic characterization of different galaxy populations (Glazebrook 2013 review) is a key observational input to distinguish between different galaxy evolutionary scenarios because it helps us to determine the number ratio of rotating disks to mergers at different cosmic epochs. This provides a way of constraining the relative role of major mergers and steady cool gas accretion in shaping galaxies, which remains a topic of discussion (e.g., Genzel et al. 2001; Tacconi et al. 2008; Dekel et al. 2009b; Förster Schreiber et al. 2009; LemoineBusserolle \& Lamareille 2010; Lemoine-Busserolle et al. 2010; Bournaud et al. 2011; Epinat 2011). Several authors have already analyzed the velocity fields and velocity dispersion maps of different galaxy samples, such as Lyman break analogs (LBAs), submm galaxies (SMGs), (U)LIRGs, $\mathrm{H} \alpha$ emitters, high- $z$ simulated spiral galaxies (SINGS) using the kinemetry methodology ${ }^{1}$ (Krajnović et al. 2006, hereafter, K06) with the aim of discerning merging and nonmerging systems on the basis of their kinematic properties (e.g., Shapiro et al. 2008; Gonçalves et al. 2010; Alaghband-Zadeh et al. 2012; Bellocchi et al. 2012; Swinbank et al. 2012; Hung et al. 2015).

The LIRG $\left(L_{\mathrm{IR}}=[8-1000 \mu \mathrm{m}]=10^{11}-10^{12} L_{\odot}\right)$ and ULIRG (ULIRGs, $L_{\mathrm{IR}}>10^{12} L_{\odot}$ ) galaxy populations are particularly relevant to the study of galaxy evolution since, although rare in the local Universe, they are far more numerous at high- $z$ and they are responsible for a significant portion of previous star formation prior to redshift $z \sim 1$ (e.g., Le Floc'h et al. 2005; PérezGonzález et al. 2005, 2008). Several authors have suggested that high- $z$ LIRGs are scaled-up versions of low- $z$ LIRGs (e.g., Pope et al. 2006; Papovich et al. 2007; Elbaz et al. 2010, 2011; Nordon et al. 2010, 2012; Takagi et al. 2010), finding that in the local Universe (U)LIRGs cover a similar SFR range than normal high$z$ SFGs (e.g., Wuyts et al. 2011). Therefore, low- $z$ (U)LIRGs offer a unique opportunity to study extreme star-forming events at high linear resolution and $\mathrm{S} / \mathrm{N}$ and compare these with those observed at high- $z$.

In this paper we present the results from applying the kinemetry method to a large sample of 38 local (U)LIRG systems (51 individual galaxies) observed with the VIMOS/VLT integral field unit (IFU). The same approach as in Bellocchi et al. (2012) is taken into account considering both locally observed and high- $z$ simulated (U)LIRGs systems. Thus, this study allows us to constrain the disk/merger fraction in the local Universe as well as to compare such a ratio with that derived for high- $z$ populations.

The paper is structured as follows. In Sect. 2, we present the sample giving details about the observations, data reductions, line fitting, and map construction. Section 3 is devoted to the description of kinemetry analysis and its potential in distinguishing disks from mergers when applying two different methods (Shapiro et al. 2008; Bellocchi et al. 2012, hereafter, S08 and B12) to a sample of local and high- $z$ simulated galaxies. Finally, our main results and conclusions are summarized in Sect. 4. Throughout the paper, we consider $H_{0}=70 \mathrm{~km} \mathrm{~s}^{-1} \mathrm{Mpc}^{-1}$, $\Omega_{\mathrm{M}}=0.3$, and $\Omega_{\Lambda}=0.7$.

\section{Observations, data reduction, and data analysis}

\subsection{Sample and morphological class}

The (U)LIRG sample analyzed in this work is the same than that analyzed in Bellocchi et al. (2013, hereafter, B13) in which the 2D kinematic properties of the ionized gas $(\mathrm{H} \alpha)$ are discussed. To summarize, it contains a total of 38 (U)LIRGs systems (51 individual galaxies) of the southern hemisphere drawn from the Revised Bright Galaxy Sample (RBGS; Sanders et al. 2003). Of these systems 31 are LIRGs (i.e., $\left\langle L_{\mathrm{IR}}\right\rangle=2.9 \times 10^{11} L_{\odot}$ ) with a mean redshift of 0.024 (corresponding to $D \sim 100 \mathrm{Mpc}$ ), and the remaining seven are ULIRGs (i.e., $\left\langle L_{\mathrm{IR}}\right\rangle=1.6 \times 10^{12} L_{\odot}$ ) with a mean redshift of $0.069(D \sim 300 \mathrm{Mpc})$; see Table A.1 and Arribas et al. (2008, hereafter, A08) for details. This sample thus includes a good representation of the relatively less-studied

\footnotetext{
Kinemetry is a tool that is able to quantify kinematic asymmetries in the velocity field and velocity dispersion maps of the systems with respect to those characterizing an ideal rotating disk. It is described in Sect. 3.
} 
LIRG luminosity range. It also encompasses a wide variety of morphological types, suggesting different dynamical phases (isolated spirals, interacting galaxies, and ongoing and post mergers), and nuclear excitations (HII, Seyfert, and LINER). Eleven out of 51 galaxies show evidence in their optical nuclear spectra of hosting an AGN, showing high [NII]/H $\alpha$ values and/or broad $\mathrm{H} \alpha$ emission lines (e.g., IRAS F07027-6011N, IRAS F05189-2524, IRAS F12596-1529, IRAS F21453-3511; see Monreal-Ibero et al. 2010; Arribas et al. 2012, 2014, hereafter, MI10, A12, A14 respectively). Most of these objects (46 out of 51) show outflows of ionized gas, studied in A14, while a smaller fraction (22 out of 40) show outflows of neutral gas, studied in Cazzoli et al. (2014, 2016). The sample is not complete either in luminosity or in distance. However, it covers the relevant luminosity range fully and is representative of the different morphologies within the (U)LIRG phenomenon (see Fig. 1).

The morphological class was derived using Digital Sky Survey (DSS) ground-based images and, when available, additional archival Hubble Space Telescope (HST) images. Except for one case, the morphological classification according to the DSS images is in good agreement with that derived via the HST images $^{2}$. The sources were morphologically classified following a simplified version of the scheme proposed by Veilleux et al. (2002) with three main classes instead of five (see RodríguezZaurín et al. 2011, hereafter, RZ11 and references therein for further details). We refer to the images published in B13 (in their Appendix A) in which the $\mathrm{H} \alpha$ kinematic maps are shown for each galaxy and to their DSS/HST images published in RZ11. Briefly, we remind the three morphological classes defined as follows:

- Class 0: objects that appear to be single isolated galaxies with a relatively symmetric disk morphology and without evidence for a strong past or ongoing interaction (hereafter, disk).

- Class 1: objects in a pre-coalescence phase with two welldifferentiated nuclei separated a projected distance $>1.5 \mathrm{kpc}$. For these objects, it is still possible to identify the individual merging galaxies and, in some cases, their corresponding tidal structures due to the interaction (hereafter, interacting).

- Class 2: objects with two nuclei separated a projected distance $\leq 1.5 \mathrm{kpc}$ or a single nucleus with a relatively asymmetric morphology suggesting a post-coalescence merging phase (hereafter, merger).

In Table A.1 we present the main properties of the sample. In some cases the properties of individual galaxies in multiple systems could be inferred separately and were therefore treated individually (see B13 for details).

\subsection{Observations and data reduction}

The observations have been described in detail in previous papers (i.e., A08, MI10, RZ11, and B13). In brief, they were carried out using the Integral Field Unit of VIMOS (Le Fèvre et al. 2003), at the Very Large Telescope (VLT), covering the spectral range (5250-7400) $\AA$ with the high resolution grating GG435 ("HR-orange" mode) and a mean spectral resolution of 3470

\footnotetext{
2 For 19 out of 38 of the systems in our sample, DSS and HST images are available. The HST image only reveals more features than those shown in the DSS image for IRAS F06206-6315, morphologically classifying this galaxy as type 1 vs. type $2 / 0$ when using HST and DSS images, respectively.
}

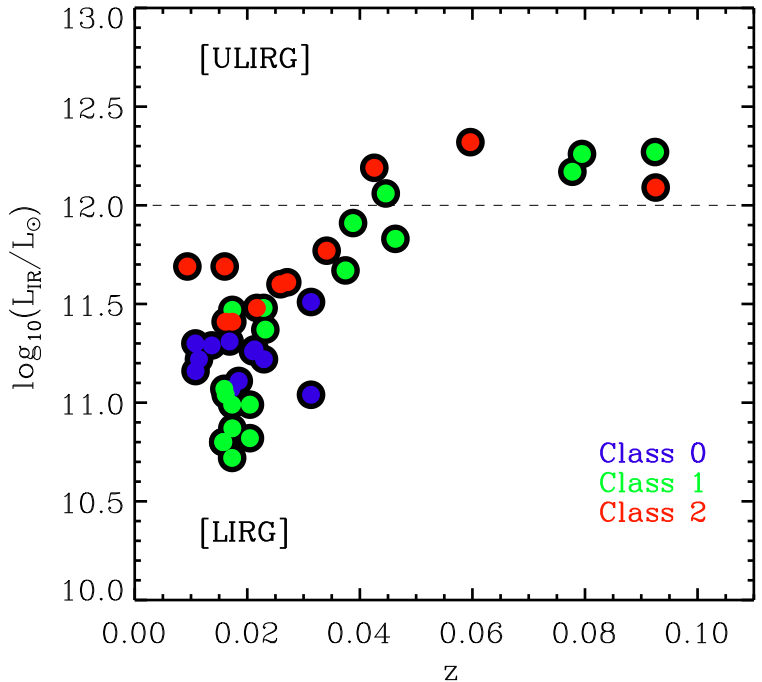

Fig. 1. Distribution of the VIMOS sample in the infrared luminosityredshift plane. The different colors represent the three stages of interactions: we show the "Class 0" single isolated objects in blue, the "Class 1 " interacting galaxies in a pre-coalescence phase in green, and the "Class 2" objects representing single systems with evidence of having suffered a merger (post-coalescence phase) in red. The horizontal dashed line separates the LIRG and ULIRG domains.

(dispersion of $0.62 \AA \mathrm{pix}^{-1}$ ). The effective field of view (FoV) in this configuration is $29.5^{\prime \prime} \times 29.5^{\prime \prime}$ with a spaxel scale of $0.67^{\prime \prime}$ per fiber (i.e., 1936 spectra are obtained simultaneously from a $44 \times 44$ fibers array).

The VIMOS data are reduced with a combination of the pipeline Esorex (versions 3.5.1 and 3.6.5) included in the pipeline provided by ESO, and different customized IDL and IRAF scripts. The basic data reduction (i.e., bias subtraction, flat field correction, spectra tracing and extraction, correction of fiber and pixel transmission, and relative flux calibration) is performed using the Esorex pipeline. The four quadrants per pointing are reduced individually and then combined into a single data cube. Then, the four independent dithered pointing positions are combined together to end up with the final "supercube", containing $44 \times 44$ spaxels for each object (i.e., 1936 spectra). For the wavelength calibration description, we refer to A08 and RZ11.

\subsection{Data analysis}

The observed $\mathrm{H} \alpha$ and [NII] $\lambda \lambda 6548,6583 \AA$ emission lines of the individual spectra are fitted to Gaussian profiles using an IDL routine (i.e., MPFITEXPR, implemented by C. B. Markwardt). This algorithm derives the best set of lines that match the available data. In case of adjusting multiple lines, the line flux ratios and wavelengths of the different lines are fixed according to the atomic physics. The widths are constrained to be equal for all the lines and greater than the instrumental contribution $\left(\sigma_{\text {INS }}\right)$. The results of the fit were presented in the Appendix A in B13, in which the $\mathrm{H} \alpha$ maps are shown (i.e., flux intensity, velocity field, and velocity dispersion maps).

As largely described in B13, narrow (or systemic) and broad components ${ }^{3}$ were identified in most of the systems to properly fit the spectra. In this analysis, we focus on the kinematic maps of the systemic component, assumed to be the

\footnotetext{
3 The distinction between narrow and broad components has been designated according to their line widths.
} 
narrow component of the emission line, since the spatial distribution and kinematic properties of this component represent those of the entire galaxy.

\subsection{Simulated high-z observations: the resolution effects}

In order to investigate how a decreasing angular resolution affects our results, we simulate observations at $z=3$ with a typical pixel scale of $0.1^{\prime \prime}$ (the same pixel scale as the IFU NIRSpec/JWST) as carried out in B12, considering resolution effects alone. Since the angular distance evolves less than $10 \%$ in the redshift range $z=2-3$, our simulated observations at $z=3$ are relevant for a direct comparison to observations at $z \sim 2$.

The "simulated" FoV of the maps ranges between $\sim 1^{\prime \prime} \times 1$ " up to $\sim 5^{\prime \prime} \times 5^{\prime \prime}$ with the scale of $\sim 7.7 \mathrm{kpc} \operatorname{arcsec}^{-1}$ assuming the $\Lambda \mathrm{CDM}$ cosmology considered in this work.

\section{Kinemetry analysis}

We investigate the power of the kinemetry methodology in studying the kinematic asymmetries in (U)LIRG systems. In particular, the same approach described in B12 (i.e., using the S08 and B12 criteria, hereafter, "unweighted" and "weighted" methods, respectively) is applied to i) the entire observed local sam$\mathrm{ple}^{4}$ and then to ii) the simulated high- $z$ kinematic maps. In this section, the results derived from applying these two methodologies are discussed.

\subsection{The method}

The kinemetry method comprises a decomposition of the moment maps into Fourier components using ellipses. We briefly describe the main steps presented in K06 to achieve a clearer understanding of this analysis.

The Fourier analysis is the most straightforward way to characterize any periodic phenomenon: the periodicity of a kinematic moment can easily be seen by expressing the moment in polar coordinates where $K(x, y) \rightarrow K(r, \psi)$. The map $K(r, \psi)$ can be expanded as follows to a finite number $(N+1)$ of harmonic terms (frequencies):

$K(r, \psi)=A_{0}(r)+\sum_{n=1}^{N} A_{n}(r) \sin (n \cdot \psi)+B_{n}(r) \cos (n \cdot \psi)$,

where $\psi$ is the azimuthal angle in the plane of the galaxy (measured from the major axis) and $r$ is the radius of a generic ellipse. The amplitude and phase coefficients $\left(k_{n}, \phi_{n}\right)$ are easily calculated from the $A_{n}, B_{n}$ coefficients as $k_{n}=\sqrt{A_{n}^{2}+B_{n}^{2}}$ and $\phi_{n}=\arctan \left(\frac{A_{n}}{B_{n}}\right)$.

For an ideal rotating disk the $B_{1}$ term dominates the velocity profile, representing the circular velocity in each ring $r$, while the $A_{0}$ term dominates the velocity dispersion profile giving the systemic velocity of each ring. Thus, higher order terms $\left(A_{n}, B_{n}\right)$ indicate deviations from symmetry. In the kinemetry analysis, we assume for each ellipse a covering factor $=0.7$, a position angle $(\Gamma)$, and a flattening $(q)$ free to vary, and the peak of the VIMOS continuum emission as the center of the ellipse.

\footnotetext{
4 The final total number of galaxies analyzed in this work with kinemetry is 50 instead of 51, since the galaxy F08424-3130 $\mathrm{N}$ is located in the edge of the VIMOS FoV and then excluded from this analysis.
}

The covering parameter represents the minimum percentage of the points along an ellipse needed to start the analysis. In our case, 0.7 means that the program stops if fewer than $70 \%$ of the points along an ellipse are not covered by data. The position angle of the velocity field $(\Gamma)$ identifies the angle where the velocity field peaks with respect to the north coordinate. The flattening $(q)$ is defined as the ratio of the semiminor $(b)$ to the semimajor (a) axes of the ellipse, i.e., $q=b / a$. When $q$ is let free to vary allows us to consider general cases, such as tilted/wrapped disks. For further details on these assumptions, see B12.

\subsection{The kinematic criteria: the "unweighted" and "weighted" methods}

We considered different kinematic criteria with the aim of distinguishing systems that have suffered recent major merger events (i.e., mergers) and those without any signs of interacting or merger activity (i.e., disks).

As a first approach we apply the S08 method ("unweighted" method) where the kinematic asymmetries of the velocity field and velocity dispersion maps are defined, respectively, as follows:

$v_{\mathrm{asym}}=\left\langle\frac{k_{\mathrm{avg}, v}}{B_{1, v}}\right\rangle_{r}, \quad \sigma_{\mathrm{asym}}=\left\langle\frac{k_{\mathrm{avg}, \sigma}}{B_{1, v}}\right\rangle_{r}$,

where $k_{\mathrm{avg}, v}=\left(k_{2, v}+k_{3, v}+k_{4, v}+k_{5, v}\right) / 4$ and $k_{\mathrm{avg}, \sigma}=\left(k_{1, \sigma}+k_{2, \sigma}+\right.$ $\left.k_{3, \sigma}+k_{4, \sigma}+k_{5, \sigma}\right) / 5$.

On the other hand, we apply the method presented in B12 ("weighted" method) as well. This revised method is based on the results that indicate that in a post-coalescence merger the inner regions are dominated by rotation while the outer parts retain larger kinematic asymmetries (e.g., Kronberger et al. 2007). With this criterion the importance of the kinematic asymmetries at larger radii is enhanced. Indeed, instead of simply averaging the asymmetries over all radii (as in S08), they are weighted according to the number of data points used in their determination. The number of data points is to first approximation proportional to the circumference of the ellipse; the circumferences $\left(\mathrm{C}_{n}\right)$ of the ellipses are computed as shown in Eq. (5) in B12. The asymmetries found in the outer ellipses contribute more significantly to the average when deriving $v_{\text {asym }}^{w}$ and $\sigma_{\text {asym }}^{w}$. We note the final formulas compute the weighted velocity and velocity dispersion asymmetries as

$v_{\mathrm{asym}}^{w}=\sum_{n=1}^{N}\left(\frac{k_{\mathrm{avg}, n}^{v}}{B_{1, n}^{v}} \cdot C_{n}\right) \cdot \frac{1}{\sum_{n=1}^{N} C_{n}}$,

$\sigma_{\mathrm{asym}}^{w}=\sum_{n=1}^{N}\left(\frac{k_{\mathrm{avg}, n}^{\sigma}}{B_{1, n}^{v}} \cdot C_{n}\right) \cdot \frac{1}{\sum_{n=1}^{N} C_{n}}$,

where $N$ is the total number of radii considered, $\mathrm{C}_{n}$ the value of the circumference for a given ellipse, the different $k_{n}\left(k_{n}^{v}\right.$ and $\left.k_{n}^{\sigma}\right)$ are the deviations concerning the velocity field and velocity dispersion maps, respectively, and $B_{1}^{v}$ is the rotational curve.

This is the first attempt in applying the kinemetry method along with kinematic criteria to a large sample local $(z<0.1)$ SFGs. This is crucial to understanding the fraction of disks and mergers locally in such systems, allowing us to compare such a ratio with those derived for high- $z$ SFGs. 


\subsection{Morphological definition of disks and mergers}

As previously described in Sect. 2, our sample consists of 50 individual galaxies covering a large $L_{\mathrm{IR}}$ range and encompasses a wide variety of morphologies, which allow us to discuss the kinematic asymmetries as a function of the galaxy properties (e.g., infrared luminosity $L_{\mathrm{IR}}$, morphological class).

We recall the Veilleux et al. (2002) classification to better interpret and discuss our data. Based on this classification system, the interacting (type 1) galaxies can be subclassified as wideor close-interacting according to their projected nuclear separation. The presence (or not) of tidal tails and/or other structures interconnecting the nuclei is considered using DSS continuum maps and, when available, HST images because they could help us to better distinguish their structure. In particular, if the nuclear projected separation is $>10 \mathrm{kpc}$, the emission of the two galaxies can be separated well in the VIMOS maps, and there is no presence of tidal tails and/or other structures between the nuclei in their continuum (DSS, HST) maps, the sources are considered wide-interacting pairs (or paired disks). A few systems (i.e., IRAS F06035-7102, IRAS F06206-6315, IRAS F125961529, IRAS F22491-1808, and IRAS F23128-5919) are classified as close-interacting pairs (or ongoing mergers), since their projected nuclear separation is smaller than $10 \mathrm{kpc}$ (but larger than $1.5 \mathrm{kpc}$ at which the limit for the coalescence phase is defined), their individual contributions cannot be disentangled in the VIMOS maps ${ }^{5}$ and they also show the presence of tidal tail structures in their continuum maps. These galaxies have a common envelope, and they are likely in a more advanced merger phase than the wide-interacting pairs. Therefore, we have distinguished four dynamical phases, where the first two are referred to as "disk", while the second two are referred to as "mergers" (case I):

$\left.\begin{array}{l}\text { - Isolated disk / class } 0 \\ \text { - Paired disk (Wide interacting pair) / class } 1 \\ {[\mathrm{~d}>10 \mathrm{kpc}]}\end{array}\right]$ 'disks'

We propose such a simplified morphological (disk-merger) classification to characterize the evolutive status of the galaxies in our sample in some way; these galaxies show a large variety of dynamical phases. In particular, as "merger" we refer to those systems where the interacting galaxies are close enough to share a common envelope and similar kinematics, which suggests they are probably going to merge. Thus, we consider these galaxies to be a single system. As "disk" we include the pure (isolated) disk galaxies as well as the "paired" galaxies which, for definition, do not share a common envelope because of their large projected separation. In this case, we cannot surely conclude that these galaxies are going to merge and, if they do, it is in a more advanced phase.

\footnotetext{
5 The galaxy IRAS F08520-6850 is considered a wide pair because the two galaxies can be well separate in the VIMOS maps, although their nuclear separation is slightly smaller than $10 \mathrm{kpc}$.
}

A similar morphological classification for the class 1 (interacting) systems was considered in A14, in which the systems were distinguished as "total system" or "individual object".

However, for the isolated disks (class 0) and postcoalescence mergers (class 2) a clearer dynamical status can be inferred with respect to that derived for the interacting systems (class 1), as a result of their diversified interaction stages, which is in between the two aforementioned classes. In this respect, we also discuss the kinemetry results derived in the case where only "isolated disks" are considered "true disks" (case II). In this case, we exclude the "paired disk" galaxies from such a group because of their supposed different dynamical status (i.e., interacting) with respect to the isolated disks. However, following our merger definition, these "paired disks" are also kept out from the "true merger" group.

\subsection{Kinematic distinction between disks and mergers}

Similarly to S08 ("unweighted" method) and the method in B12 ("weighted" method), we quantify the total kinematic asymmetry degree $K_{\text {tot }}$ of each galaxy as the combination of both the kinematic asymmetry contributions of the velocity field $\left(v_{\text {asym }}\right)$ and velocity dispersion $\left(\sigma_{\text {asym }}\right)$ maps, i.e., $K_{\text {tot }}=$ $\sqrt{\left(\sigma_{\text {asym }}^{2}+v_{\text {asym }}^{2}\right)}$ (see Eqs. (2)-(4)). We find that the ongoing merging systems have the largest kinematic asymmetry $\left(K_{\mathrm{tot}}\right)$, while isolated and paired disks and post-coalescence mergers are characterized by lower $K_{\text {tot }}$ values. In a similar way, ULIRGs show higher $K_{\text {tot }}$ with respect to LIRGs. The mean (median) $K_{\text {tot }}$ values for the different groups are shown in Table 1.

The kinematic asymmetry results (i.e., $v_{\text {asym }}$ and $\sigma_{\text {asym }}$ ) derived for the whole local sample are shown in Fig. 2 when applying the unweighted (left panels, $\left[\sigma_{\mathrm{a}}-v_{\mathrm{a}}\right]$ ) and weighted (right panels, $\left.\left[\sigma_{\mathrm{a}}^{\mathrm{w}}-v_{\mathrm{a}}^{\mathrm{w}}\right]\right)$ criteria, respectively. The same general trend is found in both the planes, where disks (isolated and paired disks) are characterized by lower kinematic asymmetries than mergers (ongoing and post-coalescence mergers).

In order to distinguish disks from mergers in the two kinematic asymmetry planes, we attempt to find out a value of the frontier applying the following approach. Since a large scatter is found in the asymmetry values of each kinematic class (i.e., disk and merger populations), the median value (instead of the mean) of each $K_{\text {tot }}$ distribution is considered (hereafter, $K_{\text {tot }}^{\text {med(disk) }}$ and $K_{\text {tot }}^{\text {med(merger) }}$ ). Then, the total kinematic asymmetry for the frontier $\left(K_{\text {tot }}^{\mathrm{F}}\right)$ is computed as the mean value of these two quantities,

$K_{\mathrm{tot}}^{\mathrm{F}}=\frac{1}{2} \times\left(K_{\mathrm{tot}}^{\mathrm{med}(\mathrm{disk})}+K_{\mathrm{tot}}^{\mathrm{med}(\text { merger })}\right)$

The uncertainty associated with this value was computed as the mean value of each median absolute deviation (or MAD) ${ }^{6}$ estimate associated with each distribution. As shown in Fig. 2, for $K_{\text {tot }} \leq K_{\text {tot }}^{\mathrm{F}}-1$ MAD no mergers are found, while for $K_{\mathrm{tot}} \geq K_{\mathrm{tot}}^{\mathrm{F}}+1$ MAD disks do not exist (dashed black frontiers). Indeed, the more disturbed objects are those classified as ongoing-mergers (i.e., IRAS F06035-7102, IRAS F06206-6315, IRAS F12596-1529, IRAS F22491-1808,

6 The respective uncertainty associated with the median value of the total kinematic asymmetry was computed as median absolute deviation (hereafter, MAD). This uncertainty returns a data set' s median absolute deviation from the median, i.e., median(|data - median(data) $\mid)$. It is a proxy for the standard deviation, but is more resistant against outliers. 

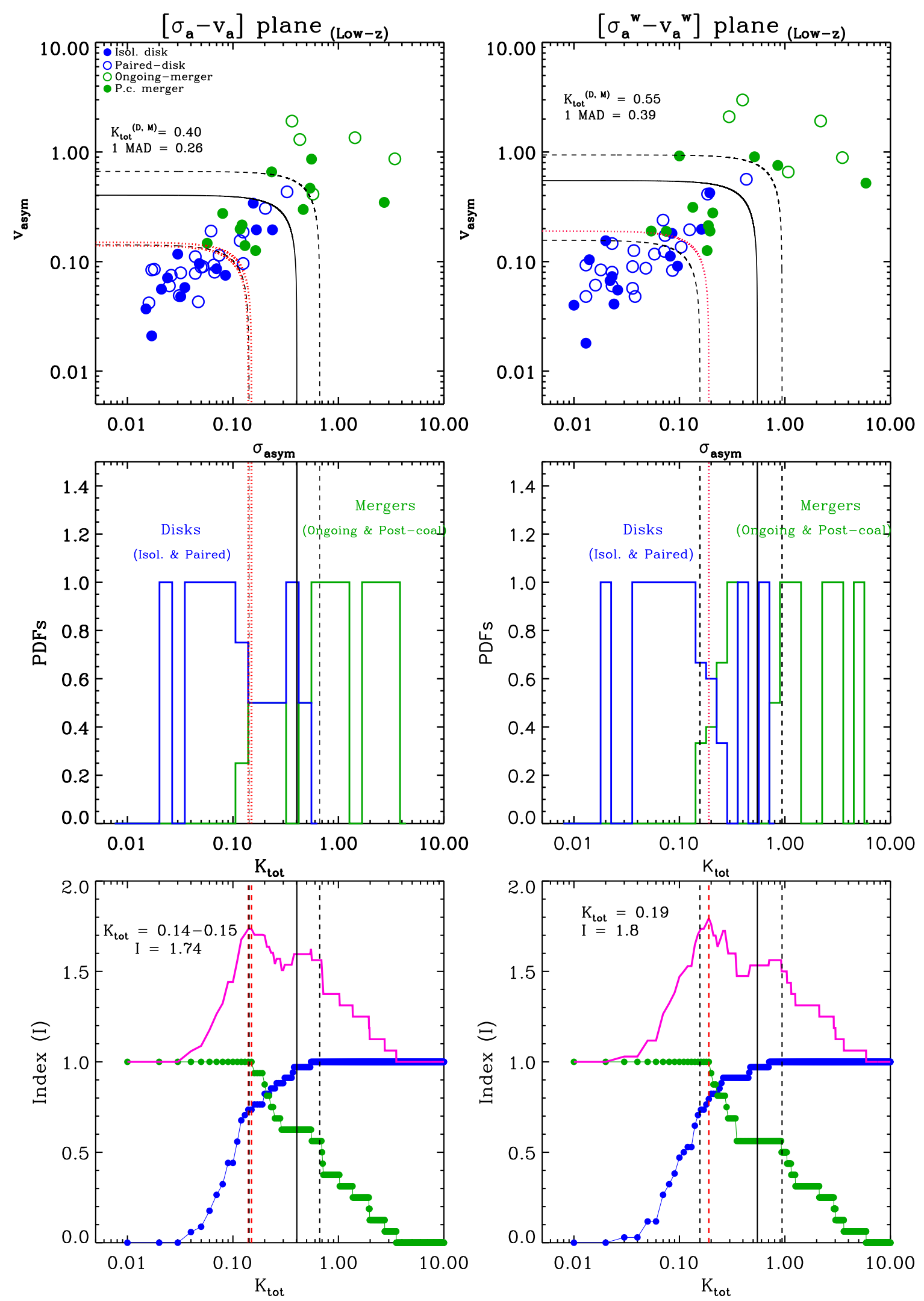

Fig. 2. Top: asymmetry measure of the velocity $v_{\text {asym }}$ and velocity dispersion $\sigma_{\text {asym }}$ fields for the whole sample observed at low- $z$ when applying the unweighted (left) or the weighted (right) methods. Different colors distinguish the different morphological types: the solid dark blue dots are the morphologically classified isolated disks, the wide pairs are in open blue dots, the post-coalescence mergers are in solid dark green dots, and the close-pairs are in open green dots. Middle: probability distribution functions (PDFs) of the respective planes normalized to the number of objects in each bin. Bottom: distribution of the total number of well classified galaxies at low- $z$ as a function of the total kinematic asymmetry $K_{\text {tot }}$ in the unweighted (left) and weighted (right) planes. In all the panels we represent the following lines: the red dashed line(s) represents the "optimal" frontier, which gives us the $K_{\mathrm{tot}}$ value for which the maximum index $I$ is derived; the solid black line indicates the $K_{\mathrm{tot}}^{\mathrm{F}}$ value derived according to Eq. (5), while the two dashed black lines represent the statistical frontiers $\left(K_{\mathrm{tot}}^{\mathrm{F}} \pm 1 \mathrm{MAD}\right)$. 
E. Bellocchi et al.: Kinematic asymmetries of (U)LIRGs

Table 1. Mean (median) $K_{\text {tot }}$ asymmetry values of the (U)LIRG sample.

\begin{tabular}{lcr}
\hline \hline Systems & $K_{\text {tot }}$ & \# Objects \\
$(1)$ & $(2)$ & $(3)$ \\
\hline class 0 (Isolated disk) & $0.13 \pm 0.03(0.11)$ & $13 / 50$ \\
class 1 (Paired disk) & $0.14 \pm 0.03(0.10)$ & $21 / 50$ \\
class 1 (Ongoing merger) & $1.9 \pm 0.47(1.95)$ & $5 / 50$ \\
class 1 (Pair disk \& Ongoing merger) & $0.48 \pm 0.16(0.12)$ & $26 / 50$ \\
class 2 (Post-coalescence merger) & $0.64 \pm 0.22(0.29)$ & $11 / 50$ \\
\hline LIRGs & $0.27 \pm 0.08(0.11)$ & $43 / 50$ \\
ULIRGs & $1.37 \pm 0.42(1.03)$ & $7 / 50$ \\
(U)LIRG & $0.43 \pm 0.10(0.16)$ & 50 \\
\hline AGN (LIRGs) & $0.43 \pm 0.25(0.22)$ & $4 / 11$ \\
AGN (ULIRGs) & $1.73 \pm 0.67(1.97)$ & $7 / 11$ \\
AGN (U)LIRG & $0.91 \pm 0.34(0.29)$ & 11 \\
\hline
\end{tabular}

Notes. Column (1): system. Column (2): mean (and median) total kinematic asymmetry. Column (3): fraction of galaxies in each subsample.

and IRAS F23128-5919) ${ }^{7}$ along with some post-coalescence sources that show high asymmetries (i.e., IRAS F05189-2524, IRAS 09022-3615, IRAS F10257-4339, and IRAS F130012339), characterized by very disturbed kinematic maps. There is a "transition region" (i.e., $K_{\text {tot }}^{\mathrm{F}}-1 \mathrm{MAD}<K_{\mathrm{tot}}<K_{\mathrm{tot}}^{\mathrm{F}}+1$ MAD) where the distinction between disk and merger is difficult. The large dispersion and overlap in the kinemetry results derived for our isolated, pre- and post-coalescence systems highlight the uncertainty in deriving a clear value of $K_{\text {tot }}$ that is able to clearly separate disks from mergers. The middle panels in the same figure show the corresponding probability distribution functions (PDFs) of each morphological class normalized to the number of objects in each bin.

Both the unweighted and weighted methods give similar results, although the weighted method allows us to distinguish disks slightly better. This is better visible if, apart from the statistical approach, we determine the "optimal" value of the frontier that is able to classify our local sources in disk and merger galaxies (dashed red frontier): to this aim, the number of wellclassified galaxies as a function of the $K_{\text {tot }}$ is derived. Since the number of disks dominates the number of mergers in our sample, we then define an "index" parameter (I) as the sum of the respective fractions of well-classified systems in each morphological class (i.e., $I_{\text {disk }}$ for disks and $I_{\text {merger }}$ for mergers). In particular, $I$ is defined in Eq. (6):

$$
\begin{aligned}
I & =I_{\mathrm{disk}}+I_{\text {merger }} \\
& =\frac{\# \text { well class disks }}{\text { total } \# \text { disks }}+\frac{\# \text { well class mergers }}{\text { total } \# \text { mergers }} .
\end{aligned}
$$

The results are shown in the bottom panels of Fig. 2 in the two cases (i.e., unweighted and weighted methods): the disk contribution is represented in blue, the merger in green, and the total in magenta. The total observed distribution allows us to determine the optimal $K_{\text {tot }}$ frontier value for which the largest portion of well-classified systems $\left(I_{\max }\right)$ is achieved: this value is also shown in the figure. Two peaks are found in each distribution, and we refer to them as main and secondary peaks. The main peak $I_{\max }$ identifies the $K_{\text {tot }}$ value(s), which classifies the high fraction of systems well, where $K_{\text {tot }}\left(I_{\max }\right) \sim 0.15$ and 0.19 in

\footnotetext{
7 These sources may appear as such either because they are actually in the early phase of merging or because the limited angular resolution of VIMOS does not allow us to separate the contribution of each galaxy.
}

the unweighted and weighted planes, respectively. As visible in the figure, the statistical $K_{\text {tot }}$ values (i.e., $K_{\text {tot }} \pm 1$ MAD) approximate well the main and secondary peaks, also defining the region where the distinction between disk and merger is difficult.

Thus, at low $-z$ the highest value of $I$ is derived when the weighted method is applied (i.e., $I_{\max }=1.8, K_{\mathrm{tot}}\left(I_{\max }\right)=0.19$ ), although it is only slightly higher than that derived in the unweighted plane $\left(I_{\max } \sim 1.7\right)$. The relative disk/merger fraction, which is derived locally according to the weighted frontier in our sample, is 27/23; this implies that the number of disks almost equals that of mergers. When considering the frontier adopted by S08 $\left(K_{\text {tot }}=0.5\right)$, the index $I$ reaches the value of $\sim 1.6$, which is clearly lower than our optimal value and close to the secondary peak. If we consider this frontier half of the post-coalescence mergers are misclassified as disks; this leads to an overestimation of the disk/merger ratio. Indeed, according to their frontier the disk/merger fraction is $40 / 10$, implying that the $80 \%$ of our objects would be classified as disks.

However, a good agreement is found between the morphology and kinematic classifications; in particular, the $I_{\text {disk }}$ and $I_{\text {merger }}$ percentages for the low- $z$ sample are $79 \%(74 \%)$ vs. $100 \%$, respectively, for the weighted (unweighted) plane when isolated and paired disks are considered "true disks".

If only isolated disk galaxies are considered "true disks", the distribution of the total number of well-classified galaxies (index $I$ ), as a function of $K_{\text {tot }}$ in the unweighted and weighted planes (Fig. 4), follows the same trend as that found in the former case (bottom panels in Figs. 2 and 3).

In Table A.2 a comparison of the different (morphological and kinematic) classifications of the (U)LIRG sample is summarized.

\subsection{Total kinematic asymmetries of high-z simulated (U)LIRGs}

We apply kinemetry to the high- $z$ simulated kinematic maps (see Sect. 2.4) and the results are shown in Fig. 3 for the unweighted (left panels, $\left[\sigma_{\mathrm{a}}-v_{\mathrm{a}}\right]_{\mathrm{High}-z}$ ) and weighted (right panels, $\left.\left[\sigma_{\mathrm{a}}^{\mathrm{w}}-v_{\mathrm{a}}^{\mathrm{w}}\right]_{\text {High-z }}\right)$ planes, respectively. As expected, the results are characterized by lower kinematic asymmetries than those obtained locally (e.g., Gonçalves et al. 2010, B12) because the kinematic deviations are smoothed as a consequence of the lowering of the linear resolution; this smoothing makes 

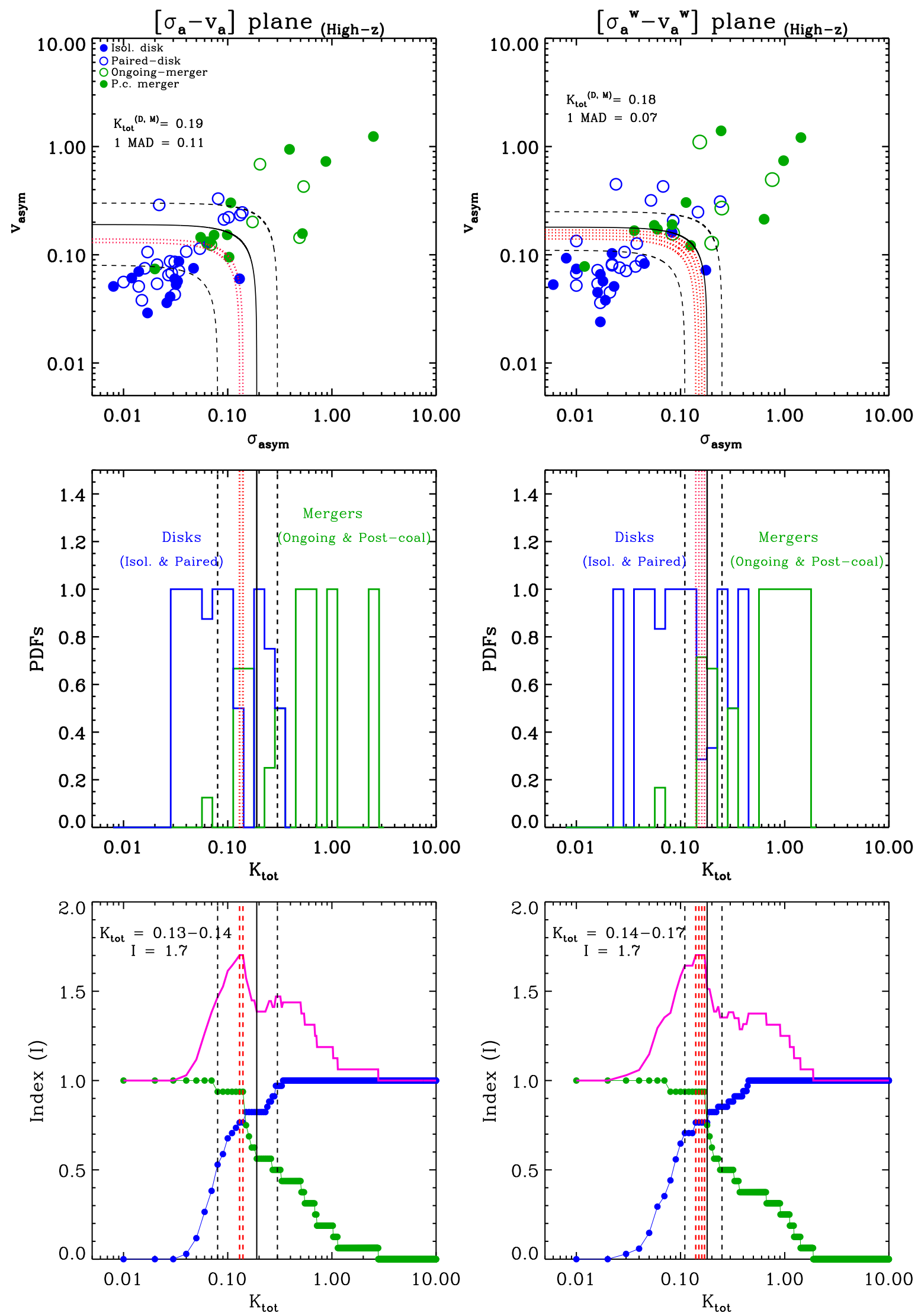

Fig. 3. Results in the $\left(\sigma_{\mathrm{a}}-v_{\mathrm{a}}\right)$ and $\left(\sigma_{\mathrm{a}}^{\mathrm{w}}-v_{\mathrm{a}}^{\mathrm{w}}\right)$ planes for the whole sample simulated at $z=3$. The panels and symbols are the same as those shown in Fig. 2. In all the panels we represent the following lines: red dashed lines represent the "optimal" frontiers, which give us the $K_{\text {tot }}$ values for which the maximum index $I$ is derived; the solid black line indicates the $K_{\mathrm{tot}}^{\mathrm{F}}$ value derived according to Eq. (5), while the two dashed black lines represent the statistical frontiers $\left(K_{\mathrm{tot}}^{\mathrm{F}} \pm 1 \mathrm{MAD}\right)$. 

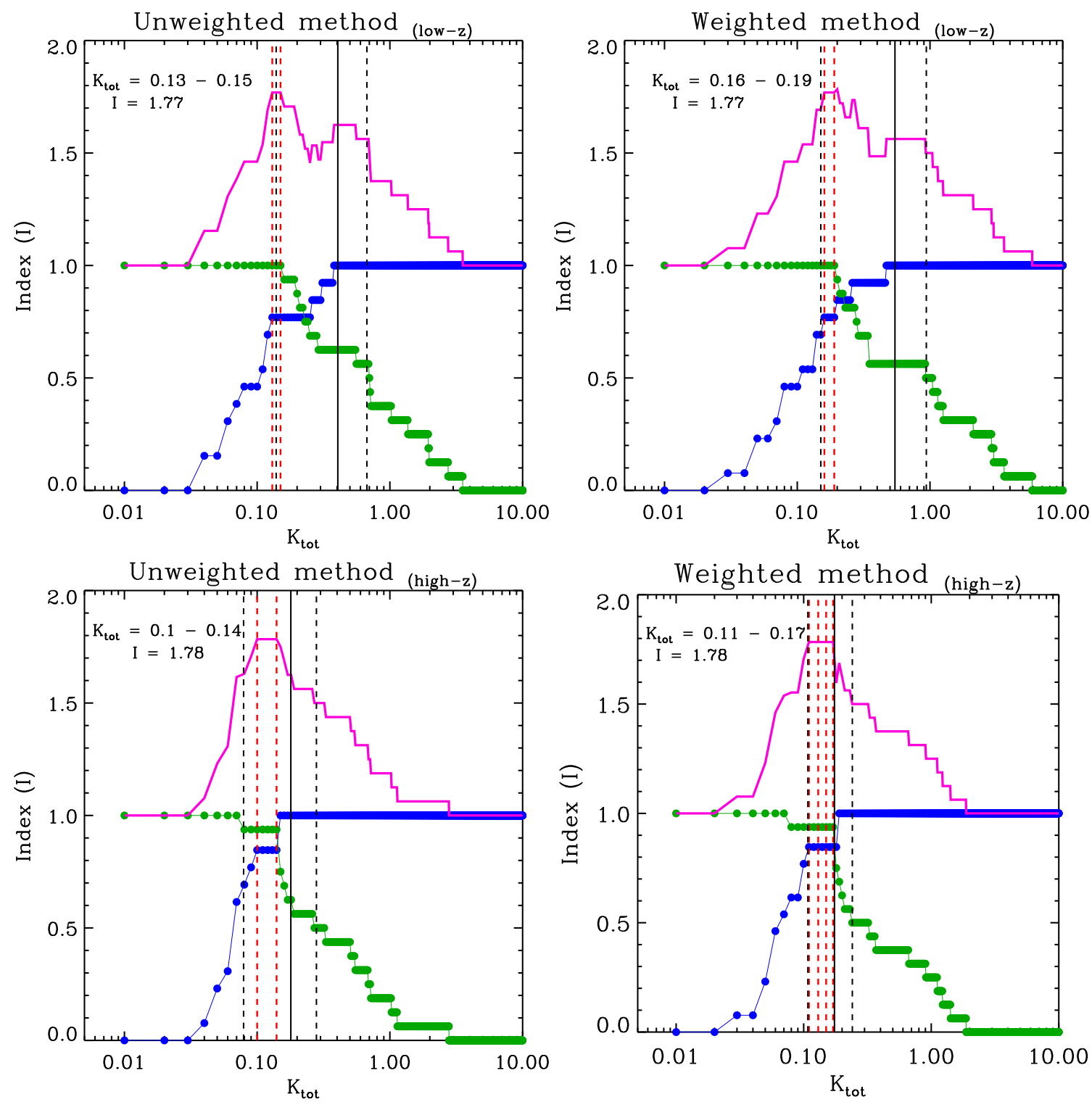

Fig. 4. Distribution of the total number of well-classified galaxies, where $I$ (magenta) equals the sum of $I_{\text {disk }}$ (blue) and $I_{\text {merger }}$ (green), at low(upper panels) and at high- $z$ (bottom panels) as a function of the total kinematic asymmetry $K_{\text {tot }}$ in the unweighted (left) and weighted (right) planes when assuming that only the isolated disks are considered "true disks" and ongoing and post-coalescence mergers are considered "true mergers". The red dashed lines represent the "optimal" frontiers, which give us the $K_{\text {tot }}$ values for which the maximum index $I$ is derived; the solid black line indicates the $K_{\mathrm{tot}}^{\mathrm{F}}$ value derived according to Eq. (5), while the two dashed black lines represent the statistical frontiers $\left(K_{\mathrm{tot}}^{\mathrm{F}} \pm 1 \mathrm{MAD}\right)$.

objects appear more symmetric than they actually are. However, a few sources among the ongoing- (i.e., IRAS F06035-7102, IRAS F2249-1808, IRAS F23128-5919) and post-coalescence (i.e., IRAS F09022-3615, IRAS F05189-2524, IRAS F102574339, IRAS F13001-2339) mergers still preserve high values.

Thus, the distribution of the number of well-classified objects as a function of the $K_{\text {tot }}$ is considered (bottom panels in Fig. 3). The main and secondary peaks are identified as well. The maximum index $I$ of 1.7 is reached in both the planes where the $K_{\text {tot }}$ assumes the (average) value of $\sim 0.14$ and $\sim 0.16$ in the unweighted and weighted planes, respectively. We derive a disk/merger ratio at high- $z$ of $26 / 24$, which is approximately the same ratio found locally. The value adopted by S08 would imply an index $I$ of $\sim 1.4$, which is clearly lower than the optimal value that we derived. In this case, about two-thirds of our mergers would be classified as disks, largely overestimating the disk/merger ratio in our sample. In such a case, the total number of disks would largely exceed the number of mergers by a factor of 7 , i.e., of 44 disks, only 7 , or $12 \%$, are mergers.

For the high- $z$ simulated sample, the derived $I_{\text {disk }}$ and $I_{\text {merger }}$ fractions result in $76 \%$ and $94 \%$ (for both the methods), respectively, when isolated and paired disks are considered "true disks". If we exclude the paired disks from the "true disk" group, similar results are derived as well (i.e., $85 \%$ and 94\%; Table 2).

\subsection{Comparison between low- and high-z kinemetry results in our (U)LIRG sample}

As a result, the comparison between local and high- $z$ results obtained using both the unweighted and weighted methods allows us to draw the following conclusions:

- At low- $z$, similar results are found for the unweighted and weighted methods, although for the weighted method disks are slightly better separated from mergers. The optimal $K_{\text {tot }}$ 
Table 2. Comparison of the values of correctly identified disks $\left(I_{\text {disk }}\right)$ and mergers $\left(I_{\text {merger }}\right)$ along with their associated $K_{\text {tot }}$ (optimal) values when using the "unweighted" and "weighted" methods at low- and at high- $z$.

\begin{tabular}{|c|c|c|c|c|c|c|}
\hline $\begin{array}{l}\text { Method } \\
\text { (1) }\end{array}$ & $\begin{array}{c}I_{\text {disk }}(\%) \\
\text { (2) }\end{array}$ & $\begin{array}{c}I_{\text {merger }}(\%) \\
(3) \\
\text { case } I]\end{array}$ & $\begin{array}{l}K_{\text {tot }} \\
\text { (4) }\end{array}$ & $\begin{array}{c}I_{\text {disk }}(\%) \\
\text { (5) }\end{array}$ & $\begin{array}{c}I_{\text {merger }}(\%) \\
(6) \\
\text { [case } I I]\end{array}$ & $\begin{array}{l}K_{\text {tot }} \\
\text { (7) }\end{array}$ \\
\hline S08 (obs) low- $z$ & $25 / 34(74)$ & $16 / 16(100)$ & $0.14-0.15$ & $10 / 13(77)$ & $16 / 16(100)$ & $0.13-0.15$ \\
\hline B12 (obs) low- $z$ & 27/34 (79) & $16 / 16(100)$ & 0.19 & $10 / 13(77)$ & $16 / 16(100)$ & $0.16-0.19$ \\
\hline S08 (sim) high- $z$ & $26 / 34(76)$ & $15 / 16(94)$ & $0.13-0.14$ & $11 / 13(85)$ & $15 / 16(94)$ & $0.1-0.14$ \\
\hline B12 (sim) high- $z$ & $26 / 34(76)$ & 15/16 (94) & $0.14-0.17$ & $11 / 13(85)$ & $15 / 16(94)$ & $0.11-0.17$ \\
\hline
\end{tabular}

Notes. All these values are computed in the cases when, as "true disks", we assume: [case I] isolated and paired disks; [case II] only isolated disks. In both the cases ongoing and post-coalescence mergers are considered "true mergers". Column (1): method used: S08 and B12 stands for "unweighted" and "weighted" methods. "obs" and "sim" stand for observed and simulated samples. Column (2): fraction of well-classified disks over total number of disks as defined in Eq. (6). when isolated and paired disks are considered "true disks". The corresponding percentage is in brackets. Column (3): fraction of well-classified mergers over total number of mergers as defined in Eq. (6). when isolated and paired disks are considered "true disks". The corresponding percentage is in brackets. Column (4): value of the total (optimal) kinematic asymmetry derived when isolated and paired disks are considered "true disks". Columns (5-7): the same values as in Cols. (2-4) when only isolated disks are considered "true disks".

value that is able to classify the largest number of objects is $~ 0.19$ : according to this result, the derived disk/merger fraction found locally is $27 / 23$ (54\% disks, $46 \%$ mergers).

- A "transition region" $\left(\left|K_{\text {tot }}-K_{\text {tot }}^{\mathrm{F}}\right|<1\right.$ MAD), where the disk and merger classification is uncertain, is found in the asymmetry plane with the total kinematic values (at low- $z$ ) in the range $0.16(0.14)<K_{\text {tot }}<0.94(0.66)$ for the weighted (unweighted) plane(s). Outside this range $\left(\left|K_{\text {tot }}-K_{\text {tot }}^{\mathrm{F}}\right| \geq 1\right.$ MAD) we are able to classify disks and mergers well.

- At high- $z$, a trend similar to that found locally is obtained but characterized by lower total kinematic asymmetries $K_{\text {tot }}$ as a consequence of the resolution effects. Slightly better results are derived when using the weighted method, in which the main peak in the $I$ distribution is better defined, with a $K_{\text {tot }} \sim$ 0.16 and a resulting disk/merger ratio of $26 / 24$ (52\% disks, $48 \%$ mergers);

- If the frontier obtained by $\mathrm{S} 08\left(K_{\mathrm{tot}}=0.5\right)$ is considered, the fraction of well-classified objects (I) would be clearly lower down to 1.6-1.4 with respect to our optimal values $(I \sim 1.8-$ 1.7) at low- and at high- $z$, respectively. The S08 limit implies that at least half of the post-coalescence mergers would be misclassified as disks, thus leading to an overestimation of the disk/merger ratio with more than $80 \%$ of the sources classified as disk.

- If only isolated disks are considered "true disks", the index distribution $I$ as a function of $K_{\text {tot }}$ in both the planes, at lowand at high- $z$, follows the same general trend as that derived when isolated and paired disks are considered "true disks". In both the cases, the derived fractions of "well-classified disks" vs. "well-classified mergers", according to the two methods, give similar results both locally ( $20 \%$ vs. $100 \%)$ and at high-z (75-85\% vs. 94\%; Table 2$)$.

\subsection{Comparison between low- and high-z kinemetry-based results in the literature}

In this section we compare the results obtained in this work with those derived in Hung et al. (2015, hereafter, H15) for an overlapped subsample of eight interacting systems ${ }^{8}$. The H15 analysis is based on the application of the unweighted and weighted

8 The overlapped subsample is composed of the following galaxies: F06076-2139 (type 1), 08355-4944 (type 2), F10038-3338 (type 2), kinemetry-based methods to a sample of local (U)LIRGs observed with the Wide Field Spectrograph (WiFeS) and artificially redshifted to $z=1.5$, degrading spatial resolution and sensitivity.

The HST and DSS images clearly show the presence of two merging galaxies in two of these systems (F06076-2139 and F12043-3140), which could not be resolved when simulated at high- $z$ in H15. Their simulated kinematic maps show a complex and irregular pattern and are classified as merger according to the B12 criteria. Excluding these two systems, our kinematic classification of the six remaining systems (eight galaxies) is in good agreement with their findings. We only find disagreement for two of these galaxies (F10257-4339 and F18093-5744 S) that are classified as "merger" and "disk", respectively, in this work. Thus, for these eight galaxies, the same disk/merger fraction $(2 / 6)$ is derived in both the works according to the frontier considered in H15 and that derived in this analysis. If we consider the results derived for our simulated subsample at $z=3$, the derived disk/merger ratio becomes 3/5. This is because F171381017 shows more ordered kinematic maps at high- $z$ than locally, and is classified as disk according to this analysis.

For this subsample, the fractions of correctly identified disks and mergers according to our analysis are $I_{\text {disk }}=2 / 3$ and $I_{\text {merger }}=5 / 5$; according to the $\mathrm{H} 15$ analysis, $I_{\text {disk }}=0 / 1, I_{\text {merger }}=$ $5 / 7$, since IRAS F17138-1017 is the only galaxy morphologically classified as isolated disk but kinematically classified as merger. Thus, a higher number of well-classified disks and mergers is derived according to our analysis (67\% and 100\%) with respect to that derived in $\mathrm{H} 15(0 \%$ and $71 \%)$.

A lower fraction of mergers at high- $z$ was also derived by Gonçalves et al. (2010): they observed a set of Lyman break analogs (LBAs) at $z \sim 0.2$ and then redshifted their sample at $z=2.2$. The loss of resolution of their simulated maps resulted in a decreasing of the fraction of mergers from low- to high- $z$, respectively, from $\sim 70 \%$ to $<30 \%$ according to the S08 limit.

The angular resolution at which a sample is observed plays a key role in classifying galaxies as disk or merger. On the one hand, the loss of angular resolution, when simulating individual galaxies at high- $z$, tends to smooth the asymmetries in their kinematic maps, making objects appear more "disky"; on the other hand, when simulating close interacting systems at high- $z$, this

F10257-4339 (type 2), F12043-3140 (type 1), F17138-1017 (type 2), F18093-5744 (type 1), and F23128-5919 (type 1). 
could result in unresolved systems that show more complicated kinematics than in resolved systems.

\subsection{The relationships between the $K_{\text {tot }}$ versus $L_{I R}, v^{*} / \sigma$ and the projected nuclear separation}

In this section the relations between the total kinematic asymmetry $K_{\text {tot }}$ and some kinematical and dynamical parameters are considered. In particular, some trends are found when considering the $K_{\text {tot }}$ as a function of the infrared luminosity $L_{\mathrm{IR}}$, the dynamical ratio $v^{*} / \sigma^{9}$, and the projected nuclear separation. Since at low- $z$ the $K_{\text {tot }}$ values for the unweighted and weighted planes are only slightly different and the same general trend is conserved, we take into account the unweighted values (i.e., $K_{\text {tot }}=$ 0.145 ) for a possible comparison with other previous works (e.g., Gonçalves et al. 2010; Alaghband-Zadeh et al. 2012; Swinbank et al. 2012).

\subsubsection{The $K_{\text {tot }}-L_{\mathrm{IR}}$ relation}

In Fig. 5 the linear trend between the total kinematic asymmetries $K_{\text {tot }}$ as a function of the infrared luminosity $L_{\mathrm{IR}}$ is shown. This plot clearly shows the (morphological and kinematical) results summarized in Table 1 . The majority of the objects with a luminosity $L_{\mathrm{IR}} \geq 11.4 L_{\odot}$ show high total kinematic asymmetries ( 19 out of 28 galaxies with $K_{\text {tot }} \gtrsim 0.14$, green dashed area) and are classified as mergers. On the other hand, most of the less luminous objects $\left(L_{\mathrm{IR}}<11.4 L_{\odot}, 15\right.$ out of 22 galaxies with $K_{\text {tot }}<0.14$, blue dashed area) have lower kinematic asymmetries and are classified as disks. Thus, the luminosity of $\log L_{\mathrm{IR}} \sim 11.4 L_{\odot}$ seems to suggest this value could be considered a threshold luminosity that is able to distinguish disks from mergers, but a sample that is complete in luminosity is needed to confirm this result.

A correlation between the morphology and the $L_{\mathrm{IR}}$ has been already found in Veilleux et al. (2002). These authors found that LIRGs are generally spirals that show a morphology much less disturbed than that shown in ULIRGs in the early phase of the interaction.

\subsubsection{The $K_{\text {tot }}-v^{*} / \sigma$ relation}

We found a clear correlation between the different phases of the merging process and mean kinematic properties inferred from the kinematic maps in our sample. In particular, isolated disks, interacting galaxies, and merging systems define a sequence of increasing mean velocity dispersion and decreasing velocity field amplitude, which is characterized by intrinsic average dynamical ratios $\left(v^{*} / \sigma\right)$ of $4.7,3.0$, and 1.8 , respectively (see B13).

In a similar way, the total kinematic asymmetry $K_{\text {tot }}$ quantifies the kinematic asymmetry degree in a galaxy with respect to the ideal rotating disk case. In Fig. 6 the relation between (unweighted) $K_{\text {tot }}$ and $v^{*} / \sigma$ for each source is considered. A (linear $\log -\log$ ) inverse trend is found; as expected, the more rotation-dominated objects $\left(v^{*} / \sigma \gtrsim 2\right)$ generally show lower values of the total kinematic asymmetries $\left(K_{\text {tot }} \lesssim 0.14\right)$ with respect to those derived for dispersion-dominated systems (i.e., $\left.v^{*} / \sigma<2, K_{\text {tot }}>0.14\right)$. In order to quantify how well the 1D parameter $v^{*} / \sigma$ classifies disks and mergers in our sample with respect to the kinemetry results, we compute the fraction

\footnotetext{
9 The $v^{*} / \sigma$ is the intrinsic dynamical ratio defined as the ratio of the intrinsic velocity shear to the mean velocity dispersion. See B13 for further details on how these parameters were computed.
}

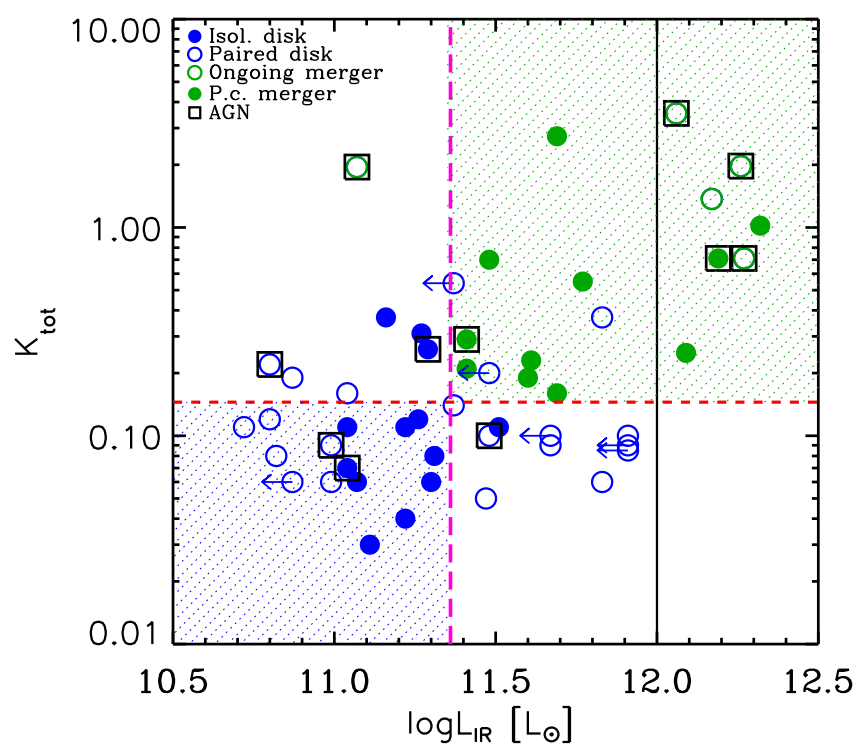

Fig. 5. Relation between the total kinematic asymmetry values, $K_{\text {tot }}$, as a function of the infrared luminosity, $L_{\mathrm{IR}}$. The vertical solid black line separates the LIRG-ULIRG domain, while the vertical dashed pink line represents the infrared luminosity value, which could separate disks from mergers. The horizontal dashed red line identifies the $K_{\mathrm{tot}}\left(I_{\max }\right)=$ 0.145 value.

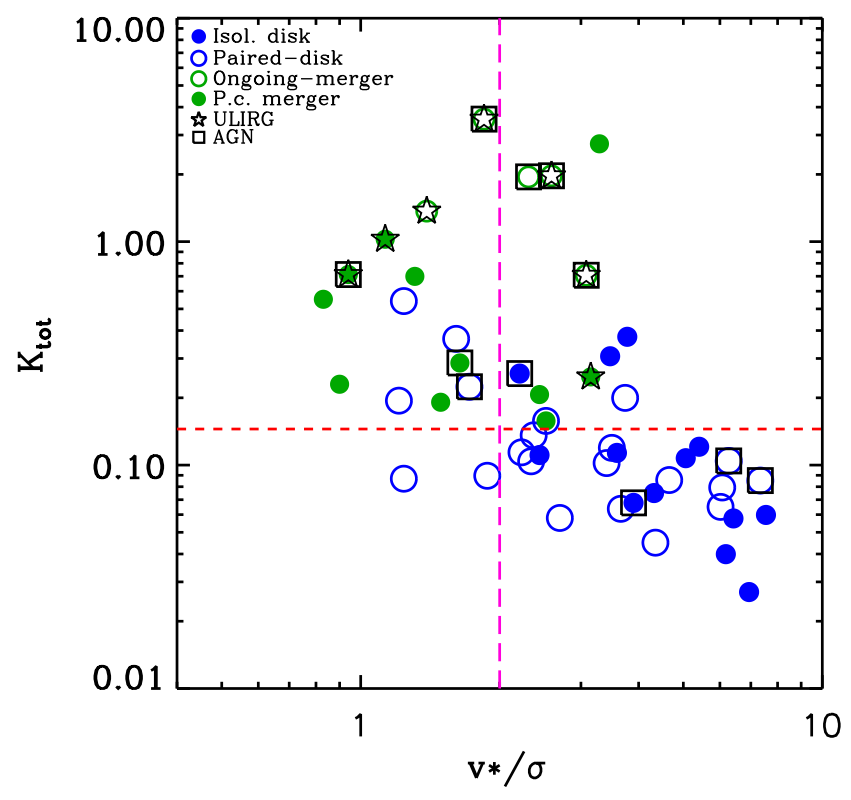

Fig. 6. Relation between the total kinematic asymmetry $K_{\text {tot }}$ and intrinsic dynamical ratio $v^{*} / \sigma$ is shown. The more rotation dominated objects $\left(v^{*} / \sigma \gtrsim 2\right)$ are those showing the lower kinematic asymmetry, while an opposite trend is found for the dispersion-dominated objects. The colors and symbols used are the same as in previous figures. Black stars represent ULIRGs, while black squares identify AGN. The horizontal dashed red line is the same as that shown in Fig. 5. The vertical dashed pink line represents the value $v^{*} / \sigma=2$.

of well-classified objects as before (i.e., Sects. 3.4 and 3.5) deriving an index $I=1.4$. This value is lower than that obtained using the total kinematic asymmetry $K_{\text {tot }}$, which is indicative that a better classification is obtained when the full 2D information is taken into account to study the kinematic asymmetries. All the ULIRGs are well classified as mergers according to our 
kinemetry frontier, while only a small fraction (3 out of 7) are classified as such according to the $v^{*} / \sigma$ parameter. This confirms the importance of the $2 \mathrm{D}$ kinematic analysis in unveiling the real status of these systems.

Our dynamical ratio threshold $\left(v^{*} / \sigma=2\right)$ is in good agreement with that derived by Kassin et al. (2012). Indeed, studying the kinematics of a large sample of 544 blue galaxies over the last $\sim 8$ billion years $(0.2<z<1.2)$, they found that such systems become progressively more ordered with time as distorted motions decrease and rotation velocities increase. They define a kinematically "settled disk" as having a ratio of ordered/random motions larger than three $(v / \sigma>3)$; they also found the fraction of settled disks increases with time (decreases with $z$ ) since $z=1.2$ for galaxies with stellar mass over $8<\log$ $M_{\star}<10.7$. The kinematic disk settling has be explained as due to: 1) a high frequency of merging at high- $z$ and 2) higher gas fraction at early times. Since both these factors decrease with time, a general kinematic settling is expected with time ("kinematic downsizing"). According to their work, the galaxies settle to become the rotation-dominated disks found in the Universe today, as the most massive galaxies are the most evolved at any time. Furthermore, at all redshifts they found that the most massive galaxies are on average the most kinematically settled while the least massive galaxies are the least kinematically settled.

In our analysis we derived a similar trend: the most massive $\left(\log \left\langle M_{\text {cdyn }}\right\rangle=10.71 M_{\odot}\right.$, median value $\left.10.69 M_{\odot}\right)$ and (morphologically) regular objects (class 0 isolated galaxies) show the highest dynamical ratio $\left(v^{*} / \sigma=4.7\right)$ while the less massive preand post-coalescence galaxies $\left(\log \left\langle M_{\text {dyn }}\right\rangle=10.68 M_{\odot}\right.$, median value $10.54 M_{\odot}$ and $10.67 M_{\odot}$, median value $10.23 M_{\odot}$, respectively; see Table 2 in B13) are characterized by lower $v^{*} / \sigma$, of 3.0 and 1.8 , respectively.

If we apply a threshold value of $v^{*} / \sigma=3$ to our data (see Fig. 5), it also suggests a good frontier to distinguish our systems in disks and mergers. In such a case, 21 out of 34 objects are well classified as disks, while 13 out of 16 are well classified as mergers, deriving an index parameter $I=1.43$. This value is higher than that derived when using the $v^{*} / \sigma=2$, but still lower with respect to that derived when using kinemetry, which gives the highest number of well-classified disks and mergers.

\subsubsection{Kinematic asymmetries as a function of the nuclear separation along the merger process}

We analyze the relation between the projected nuclear separation and total kinematic asymmetry $K_{\text {tot }}$ for those pairs of galaxies for which a nuclear separation can be computed. In particular, the nuclear separation can be estimated for 19 systems ${ }^{10}$. We computed an upper limit for the majority of the type 2 galaxies, assuming a nuclear separation that is smaller than the resolution element of the image considered. We used the HST and VIMOS continuum images to derive the projected nuclear separation. In three cases (i.e., IRAS F01341-3735, IRAS F09437+0317, IRAS F14544-4255) the DSS images were used since a larger FoV was needed to cover the whole system.

In Fig. 7 the mean value of the (unweighted) $K_{\text {tot }}\left(\left\langle K_{\text {tot }}\right\rangle\right)$ of each system is related to its nuclear projected separation. This

\footnotetext{
${ }^{10}$ We refer to the type 0 system IRAS F07027-6011, type 1 systems IRAS F01159-4443, IRAS F01341-3735, IRAS F06035-7102, IRAS F06076-2139, IRAS F06206-6315, IRAS F06259-4780, IRAS 084243130, IRAS F08520-6850, IRAS F09437+0317, IRAS F12043-3140, IRAS 12596-1529, IRAS F14544-4255, IRAS F18093-5744, IRAS F22491-1808, IRAS F23128-5919, and to a few type 2 objects IRAS 08355-4944, IRAS F10038-3338, and IRAS F21453-3511.
}

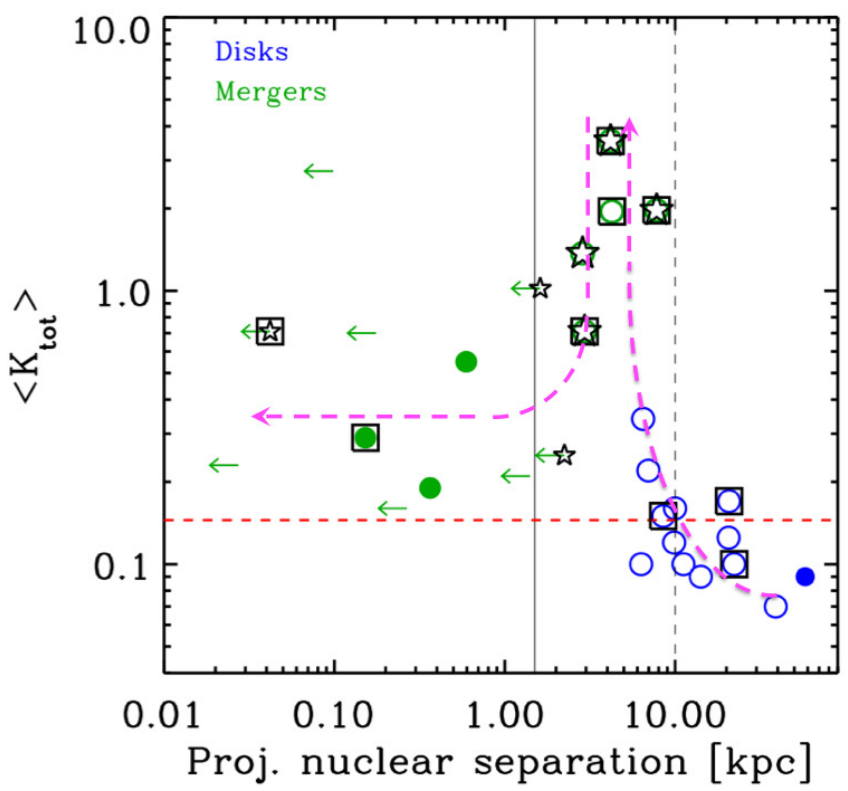

Fig. 7. Relation between the mean total kinematic asymmetry $K_{\text {tot }}$ and the nuclear projected separation in (wide and close) interacting and merging systems. The $K_{\text {tot }}$ of a system generally increases with the decreasing of the nuclear separation: it reaches its maximum value in ongoing (pre-coalescence) mergers, then decreases for post-coalescence mergers. The colors and symbols used are the same as in the previous figure (Fig. 6). The pink dashed line helps to highlight the evolutionary trend found when a galaxy goes from the wide-interacting phase to the post-coalescence phase. The horizontal dashed red line is the same as that shown in Fig. 5.

plot highlights the fact that, during the first phases of the merging process, the smaller the nuclear separation of a system the higher its total kinematic asymmetry. Moreover, the maximum asymmetry value $K_{\text {tot }}$ is reached for the ongoing mergers with nuclear separation $\sim 2-5 \mathrm{kpc}$, in which the merger phase is currently taking place. Then, a more unclear trend is shown for the post-coalescence mergers (i.e., type 2; nuclear separation less than $1.5 \mathrm{kpc}$ ), although their values are generally lower than those characterizing ongoing mergers. The virialization of the inner parts of these objects explains such results. The pink dashed line in Fig. 7 helps to highlight the possible average evolutionary sequence obtained when a galaxy goes through the wideinteracting to the post-coalescence phases.

As shown in this plot, a trend is found between the interaction stage (based on a morphological classification) and the kinematic asymmetries of our systems. Our results are in agreement with those presented in H15 (Fig. 3 in their work), where the fraction of disk/merger galaxies classified using the kinemetry criteria is shown as a function of the interaction stage. The agreement between our and their results can be explained as follows. A galaxy with a low value of the total kinematic asymmetry $K_{\text {tot }}$ $(<0.16)$ results in a disk-like (regular) kinematics while a high $K_{\text {tot }}$ value $(>0.9)$ corresponds to a more disturbed and complex kinematics. Thus, $K_{\text {tot }}$ can be considered a proxy of the fraction of mergers. In their work, going through the different interaction stages, from isolated to post-coalescence objects (from S to M4 stages), the maximum merger fraction is reached in the case of merged galaxies, which still show two distinct nuclei (M3): their M3 galaxies correspond to our close-interacting systems, which show the highest $K_{\text {tot }}$ values. In particular, the mean $K_{\text {tot }}$ values (see Table 1) as a function of the interaction stage (isolated and 
paired disks, ongoing- and post-coalescence mergers) reproduce a trend similar to that derived by $\mathrm{H} 15$.

\section{Summary}

We have carried out a kinemetry-based classification for a large sample of 38 local $(z<0.1)$ (U)LIRG systems observed with VIMOS/VLT with IFS. The sample covers a wide range of morphological types (i.e., spirals, interacting systems, and merger remnants) and it is therefore suited to study how the kinemetrybased criteria are able to distinguish disks and mergers in our objects. The disk/merger fraction allows us to constrain different evolutionary scenarios. Specifically, we applied the S08 ("unweighted") and B12 ("weighted") criteria to derive the total kinematic asymmetry $\left(K_{\text {tot }}\right)$ to our observed sample as well as to simulated data, "redshifting" our sample at $z=3$. From our analysis, we draw the following conclusions:

\subsection{Kinematic distinction between disks and mergers}

The kinematic properties derived using the kinemetry-based methods are consistent with their morphological classification. The results obtained using the weighted and unweighted methods are similar when the whole sample is considered.

We can distinguish our sample in three kinematic groups, according to the total kinematic asymmetry value $K_{\text {tot }}$, when using the weighted (unweighted) method: 1) 25 out of 50 galaxies are kinematically classified as disk with a $K_{\text {tot }} \leq 0.16(0.14)$; 2) 9 out of 50 galaxies are kinematically classified as merger with a $K_{\text {tot }} \geq 0.94(0.66)$; 3) 16 out of 50 galaxies lie in the "transition region", in which disks and mergers coexist, with 0.16 $(0.14)<K_{\text {tot }}<0.94(0.66)$.

The $K_{\text {tot }}$ frontier value that better classifies the highest numbers of disks and mergers, according to the morphology is $K_{\text {tot }}=$ $0.19(\sim 0.15)$; we obtain 27 (25) disks and 23 (25) mergers according to this value. The percentages of "correctly identified" disks and mergers at low- $z$ result in $79 \%(74 \%)-100 \%$, respectively. If only isolated disk galaxies are considered "true disks", similar fractions are obtained with both the methods.

When we apply our criteria to our systems simulated at $z=3$ just considering resolution effects, a lower total kinematic asymmetry frontier $\left(K_{\mathrm{tot}} \sim 0.16(\sim 0.14)\right)$ with respect to that found locally is derived when using the weighted (unweighted) method. We obtain 26 disks and 24 mergers according to this value.

However, the "correctly identified" disks and merger fractions for the simulated high- $z$ objects is $76 \%-94 \%$ with both the methods. If only isolated disk galaxies are considered "true disks", these values become $85 \%$ and $94 \%$, respectively. The loss of angular resolution makes objects appear more kinematically regular ("disky") than they actually are as a consequence of the smearing of the kinematic features.

\subsection{Relationships between the kinemetry-based $K_{\text {tot }}$ and morpho-kinematic parameters}

A trend is found between the $K_{\text {tot }}$ and the infrared luminosity $L_{\mathrm{IR}}$, with the most luminous objects (ULIRGs) showing the highest total kinematic asymmetries. Furthermore, the luminosity value $\log L_{\mathrm{IR}} \sim 11.4 L_{\odot}$ suggests that it could be considered a threshold value that is able to separate these two morphological classes. To confirm this, a sample complete in luminosity is needed.
An inverse trend is derived between the $K_{\text {tot }}$ and the intrinsic dynamical ratio $v^{*} / \sigma$ : morphologically classified disks show higher dynamical ratio $\left(v^{*} / \sigma \gtrsim 2\right)$ and lower total kinematic asymmetry $K_{\text {tot }}(\lesssim 0.14)$. In contrast, for the mergers $v^{*} / \sigma$ is lower $(<2)$ while $K_{\text {tot }}$ is higher $(>0.14)$. Our results support the "kinematic downsizing" scenario proposed by Kassin et al. (2012), where systems become progressively more ordered with time as distorted motions decrease and rotation velocities increase, where the most massive galaxies are on average the most kinematically settled.

An interesting trend is also found between the $K_{\text {tot }}$ and the projected nuclear separation (as a proxy of the galaxy interaction stage) along the merger process. The smaller the nuclear separation the larger the $K_{\text {tot }}$, which reaches its maximum value during the "ongoing merging phase" (nuclear separation between the galaxies of $2-5 \mathrm{kpc}$ ) and then decreases during the postcoalescence merging phase, although with a relatively large dispersion. Our results are in agreement with those derived in Hung et al. (2015), who found that the merger fraction (as a proxy of the $K_{\text {tot }}$ parameter) shows a strong trend with the galaxy interaction stage.

\subsection{The robustness of the $K_{\text {tot }}$ frontier determination in classifying disks and mergers}

From our results, the kinematic frontier we derive to distinguish disks from mergers is determined well. Indeed, when type 1 (interacting) objects are included (case I) or not (case II) in the "disk" group, the derived fractions of "well-classified" disks and merger in both the cases are very akin, i.e., $\sim 80 \%$ and $\sim 100 \%$, respectively. This result can also confirm that the "paired disk" objects can actually be considered disks according to their kinematic asymmetries.

The $K_{\text {tot }}$ limit derived by Shapiro et al. (2008) $\left(K_{\text {tot }}=0.5\right)$ to separate disks from mergers at high- $z$ is $\sim 65 \%$ larger than our $K_{\text {tot }}$ limit for the whole sample (observed locally and simulated at high- $z$ ) with both the weighted and unweighted methods. The use of this frontier would imply that the number of "disks" in our sample would be largely overestimated (classifying the $85 \%$ of the galaxies as disk), since only the ongoing- and some of the post-coalescence mergers with more complex kinematics would be classified as mergers. This, together with the effects of resolution on high- $z$ samples, suggests that the fraction of disks at high- $z$ inferred from similar kinematic criteria may be overestimated.

The value of the frontier derived using the kinemetry-based methods strongly depends on the morphological classification, which is important when analyzing high- $z$ SGFs. Such systems may be dominated by several mechanisms and characterized by different gas and dust content, stellar mass, and interaction stage. Thus, the combination of high resolution morphology (such as those coming from HST or AO-assisted imaging) along with spatially resolved kinematics will allow future research to reveal the dynamical state of such systems (Neichel et al. 2008). Multiwavelength morphological observations are needed to study the molecular gas phase (closely related to star formation), as well as the stars, to better constrain the disk/merger fraction of SFGs at high- $z$.

Acknowledgements. We acknowledge the anonymous referee for useful comments and suggestions that helped us to improve the quality and presentation of the paper. This work was funded in part by the Marie Curie Initial Training Network ELIXIR of the European Commission under contract PITN-GA-2008-214227. This work has been supported by the Spanish 
Ministry of Science and Innovation (MICINN) under grant ESP2007-65475C02-01, PNAYA2010-21161-C02-01, AYA2010-21697-C05-01, PNAYA201232295, PNAYA2012-39408-C02-01. Based on observations carried out at the European Southern Observatory, Paranal (Chile), Programs 076.B-0479(A), 078.B-0072(A), and 081.B-0108(A). This research made use of the NASA/IPAC Extragalactic Database (NED), which is operated by the Jet Propulsion Laboratory, California Institute of Technology, under contract with the National Aeronautic and Space Administration.

\section{References}

Alaghband-Zadeh, S., Chapman, S. C., Swinbank, A. M., et al. 2012, MNRAS, 424, 2232

Arribas, S., Colina, L., Monreal-Ibero, A., et al. 2008, A\&A, 479, 687

Arribas, S., Colina, L., Alonso-Herrero, A., et al. 2012, A\&A, 541, A20

Arribas, S., Colina, L., Bellocchi, E., Maiolino, R., \& Villar-Martín, M. 2014, A\&A, 568, A14

Baugh, C. M. 2006, Rep. Progr. Phys., 69, 3101

Bellocchi, E., Arribas, S., \& Colina, L. 2012, A\&A, 542, A54

Bellocchi, E., Arribas, S., Colina, L., \& Miralles-Caballero, D. 2013, A\&A, 557 A59

Bouché, N., Dekel, A., Genzel, R., et al. 2010, ApJ, 718, 1001

Bournaud, F., Daddi, E., Elmegreen, B. G., et al. 2008, A\&A, 486, 741

Bournaud, F., Chapon, D., Teyssier, R., et al. 2011, ApJ, 730, 4

Burkert, A., Genzel, R., Bouché, N., et al. 2010, ApJ, 725, 2324

Cazzoli, S., Arribas, S., Colina, L., et al. 2014, A\&A, 569, A14

Cazzoli, S., Arribas, S., Maiolino, R., \& Colina, L. 2016, A\&A, 590, A125

Ceverino, D., Dekel, A., \& Bournaud, F. 2010, MNRAS, 404, 2151

Cole, S., Aragon-Salamanca, A., Frenk, C. S., Navarro, J. F., \& Zepf, S. E. 1994, MNRAS, 271, 781

Cole, S., Lacey, C. G., Baugh, C. M., \& Frenk, C. S. 2000, MNRAS, 319, 168

Combes, F. 2004, in Penetrating Bars Through Masks of Cosmic Dust, eds. D. L. Block, I. Puerari, K. C. Freeman, R. Groess, \& E. K. Block, Astrophys. Space Sci. Lib., 319, 57

Conselice, C. J. 2006, MNRAS, 373, 1389

Conselice, C. J., Chapman, S. C., \& Windhorst, R. A. 2003, ApJ, 596, L5

Cresci, G., Hicks, E. K. S., Genzel, R., et al. 2009, ApJ, 697, 115

Cresci, G., Mannucci, F., Maiolino, R., et al. 2010, Nature, 467, 811

Daddi, E., Dickinson, M., Morrison, G., et al. 2007, ApJ, 670, 156

Dasyra, K. M., Yan, L., Helou, G., et al. 2008, ApJ, 680, 232

Davé, R. 2008, MNRAS, 385, 147

Dekel, A., Birnboim, Y., Engel, G., et al. 2009a, Nature, 457, 451

Dekel, A., Sari, R., \& Ceverino, D. 2009b, ApJ, 703, 785

Elbaz, D., Daddi, E., Le Borgne, D., et al. 2007, A\&A, 468, 33

Elbaz, D., Hwang, H. S., Magnelli, B., et al. 2010, A\&A, 518, L29

Elbaz, D., Dickinson, M., Hwang, H. S., et al. 2011, A\&A, 533, A119

Epinat, B. 2011, in Tracing the Ancestry of Galaxies, eds. C. Carignan, F. Combes, \& K. C. Freeman, IAU Symp., 277, 128

Epinat, B., Contini, T., Le Fèvre, O., et al. 2009, A\&A, 504, 789

Flores, H., Hammer, F., Puech, M., Amram, P., \& Balkowski, C. 2006, A\&A, 455,107

Förster Schreiber, N. M., Genzel, R., Lehnert, M. D., et al. 2006, ApJ, 645, 1062

Förster Schreiber, N. M., Genzel, R., Bouché, N., et al. 2009, ApJ, 706, 1364

Förster Schreiber, N. M., Shapley, A. E., Genzel, R., et al. 2011, ApJ, 739, 45

Genel, S., Genzel, R., Bouché, N., et al. 2008, ApJ, 688, 789

Genzel, R., Tacconi, L. J., Rigopoulou, D., Lutz, D., \& Tecza, M. 2001, ApJ, 563,527

Genzel, R., Burkert, A., Bouché, N., et al. 2008, ApJ, 687, 59
Genzel, R., Newman, S., Jones, T., et al. 2011, ApJ, 733, 101

Glazebrook, K. 2013, PASA, 30, 56

Gnerucci, A., Marconi, A., Cresci, G., et al. 2011, A\&A, 528, A88

Gonçalves, T. S., Basu-Zych, A., Overzier, R., et al. 2010, ApJ, 724, 1373

Governato, F., Willman, B., Mayer, L., et al. 2007, MNRAS, 374, 1479

Governato, F., Brook, C. B., Brooks, A. M., et al. 2009, MNRAS, 398, 312

Hammer, F., Flores, H., Puech, M., et al. 2009, A\&A, 507, 1313

Hopkins, P. F., Cox, T. J., Younger, J. D., \& Hernquist, L. 2009a, ApJ, 691, 1168

Hopkins, P. F., Hernquist, L., Cox, T. J., Keres, D., \& Wuyts, S. 2009b, ApJ, 691, 1424

Hung, C.-L., Rich, J. A., Yuan, T., et al. 2015, ApJ, 803, 62

Jones, T., Ellis, R., Jullo, E., \& Richard, J. 2010, ApJ, 725, L176

Kartaltepe, J. S., Sanders, D. B., Le Floc'h, E., et al. 2010, ApJ, 721, 98

Kartaltepe, J. S., Dickinson, M., Alexander, D. M., et al. 2012, ApJ, 757, 23

Kassin, S. A., Weiner, B. J., Faber, S. M., et al. 2012, ApJ, 758, 106

Kereš, D., Katz, N., Weinberg, D. H., \& Davé, R. 2005, MNRAS, 363, 2

Krajnović, D., Cappellari, M., de Zeeuw, P. T., \& Copin, Y. 2006, MNRAS, 366, 787

Kronberger, T., Kapferer, W., Schindler, S., \& Ziegler, B. L. 2007, A\&A, 473, 761

Le Fèvre, O., Saisse, M., Mancini, D., et al. 2003, in SPIE Conf. 4841, eds. M. Iye, \& A. F. M. Moorwood, 1670

Le Floc'h, E., Papovich, C., Dole, H., et al. 2005, ApJ, 632, 169

Lehnert, M. D., Nesvadba, N. P. H., Le Tiran, L., et al. 2009, ApJ, 699, 1660

Lemoine-Busserolle, M., \& Lamareille, F. 2010, MNRAS, 402, 2291

Lemoine-Busserolle, M., Bunker, A., Lamareille, F., \& Kissler-Patig, M. 2010, MNRAS, 401, 1657

Lotz, J. M., Jonsson, P., Cox, T. J., \& Primack, J. R. 2008, MNRAS, 391, 1137

Mihos, J. C., \& Hernquist, L. 1996, ApJ, 464, 641

Monreal-Ibero, A., Arribas, S., Colina, L., et al. 2010, A\&A, 517, A28

Neichel, B., Hammer, F., Puech, M., et al. 2008, A\&A, 484, 159

Noeske, K. G., Weiner, B. J., Faber, S. M., et al. 2007, ApJ, 660, L43

Nordon, R., Lutz, D., Shao, L., et al. 2010, A\&A, 518, L24

Nordon, R., Lutz, D., Genzel, R., et al. 2012, ApJ, 745, 182

Ocvirk, P., Pichon, C., \& Teyssier, R. 2008, MNRAS, 390, 1326

Papovich, C., Rudnick, G., Le Floc'h, E., et al. 2007, ApJ, 668, 45

Pérez-González, P. G., Rieke, G. H., Egami, E., et al. 2005, ApJ, 630, 82

Pérez-González, P. G., Rieke, G. H., Villar, V., et al. 2008, ApJ, 675, 234

Pope, A., Scott, D., Dickinson, M., et al. 2006, MNRAS, 370, 1185

Puech, M. 2010, MNRAS, 406, 535

Puech, M., Hammer, F., Hopkins, P. F., et al. 2012, ApJ, 753, 128

Robertson, B., Bullock, J. S., Cox, T. J., et al. 2006, ApJ, 645, 986

Rodríguez-Zaurín, J., Arribas, S., Monreal-Ibero, A., et al. 2011, A\&A, 527, A60

Sanders, D. B., \& Mirabel, I. F. 1996, ARA\&A, 34, 749

Sanders, D. B., Mazzarella, J. M., Kim, D.-C., Surace, J. A., \& Soifer, B. T. 2003, AJ, 126, 1607

Shapiro, K. L., Genzel, R., Förster Schreiber, N. M., et al. 2008, ApJ, 682, 231

Somerville, R. S., Primack, J. R., \& Faber, S. M. 2001, MNRAS, 320, 504

Swinbank, A. M., Sobral, D., Smail, I., et al. 2012, MNRAS, 426, 935

Tacconi, L. J., Neri, R., Chapman, S. C., et al. 2006, ApJ, 640, 228

Tacconi, L. J., Genzel, R., Smail, I., et al. 2008, ApJ, 680, 246

Takagi, T., Ohyama, Y., Goto, T., et al. 2010, A\&A, 514, A5

van Starkenburg, L., van der Werf, P. P., Franx, M., et al. 2008, A\&A, 488, 99

Veilleux, S., Kim, D.-C., \& Sanders, D. B. 2002, ApJS, 143, 315

Wisnioski, E., Glazebrook, K., Blake, C., et al. 2011, MNRAS, 417, 2601

Wright, E. L. 2006, PASP, 118, 1711

Wuyts, S., Förster Schreiber, N. M., van der Wel, A., et al. 2011, ApJ, 742, 96

Yang, Y., Flores, H., Hammer, F., et al. 2008, A\&A, 477, 789

Zamojski, M., Yan, L., Dasyra, K., et al. 2011, ApJ, 730, 125 


\section{Appendix A: Additional tables}

Table A.1. General properties of the (U)LIRG sample.

\begin{tabular}{|c|c|c|c|c|c|c|c|}
\hline $\begin{array}{l}\text { ID1 } \\
\text { IRAS } \\
(1)\end{array}$ & $\begin{array}{l}\text { ID2 } \\
\text { Other } \\
(2)\end{array}$ & (3) & $\begin{array}{c}D \\
(\mathrm{Mpc}) \\
(4)\end{array}$ & $\begin{array}{c}\text { Scale } \\
\left(\mathrm{pc} /{ }^{\prime \prime}\right) \\
(5)\end{array}$ & $\begin{array}{c}\log L_{\mathrm{IR}} \\
\left(L_{\odot}\right) \\
(6)\end{array}$ & $\begin{array}{c}\text { Class } \\
(7)\end{array}$ & Notes \\
\hline F01159-4443 S & ESO 244-G012 & 0.022903 & 99.8 & 462 & - & 1 & $\mathrm{a}, \mathrm{d}$ \\
\hline F01159-4443 N & ESO 244-G012 & 0.022903 & 99.8 & 462 & 11.48 & 1 & $\mathrm{a}, \mathrm{d}$ \\
\hline F01341-3735 S & ESO 297-G012 & 0.017305 & 75.1 & 352 & 10.72 & 1 & $\mathrm{a}, \mathrm{d}$ \\
\hline F01341-3735 N & ESO 297-G011 & 0.017305 & 75.1 & 352 & 10.99 & 1 & $\mathrm{a}, \mathrm{d}$ \\
\hline F04315-0840 & NGC 1614 & 0.015983 & 69.1 & 325 & 11.69 & 2 & \\
\hline F05189-2524 & & 0.042563 & 188.2 & 839 & 12.19 & 2 & \\
\hline F06035-7102 & & 0.079465 & 360.7 & 1501 & 12.26 & 1 & \\
\hline F06076-2139 S & & 0.037446 & 165 & 743 & - & 1 & $\mathrm{a}, \mathrm{d}$ \\
\hline F06076-2139 N & & 0.037446 & 165 & 743 & 11.67 & 1 & $\mathrm{a}, \mathrm{d}$ \\
\hline F06206-6315 & & 0.092441 & 423.3 & 1720 & 12.27 & 1 & \\
\hline F06259-4780 S & ESO 255-IG007 & 0.038790 & 171.1 & 769 & - & 1 & $\mathrm{~b}, \mathrm{~d}$ \\
\hline F06259-4780 C & ESO 255-IG007 & 0.038790 & 171.1 & 769 & - & 1 & $\mathrm{~b}, \mathrm{~d}$ \\
\hline F06259-4780 N & ESO 255-IG007 & 0.038790 & 171.1 & 769 & 11.91 & 1 & $\mathrm{~b}, \mathrm{~d}$ \\
\hline F06295-1735 & ESO 557-G002 & 0.021298 & 92.7 & 431 & 11.27 & 0 & \\
\hline F06592-6313 & & 0.022956 & 100 & 464 & 11.22 & 0 & \\
\hline F07027-6011 S & AM 0702-601 & 0.031322 & 137.4 & 626 & 11.51 & 0 & $\mathrm{a}, \mathrm{d}$ \\
\hline F07027-6011 N & AM 0702-601 & 0.031322 & 137.4 & 626 & 11.04 & 0 & $\mathrm{a}, \mathrm{d}$ \\
\hline F07160-6215 & NGC 2369 & 0.010807 & 46.7 & 221 & 11.16 & 0 & \\
\hline $08355-4944$ & & 0.025898 & 113.1 & 521 & 11.60 & 2 & \\
\hline 08424-3130 S & ESO 432-IG006 & 0.016165 & 70.1 & 329 & 11.04 & 1 & $\mathrm{a}, \mathrm{d}$ \\
\hline $08424-3130 \mathrm{~N}$ & ESO 432-IG006 & 0.016165 & 70.1 & 329 & - & 1 & $\mathrm{a}, \mathrm{d}$ \\
\hline F08520-6850 E & ESO 60-IG016 & 0.046315 & 205.4 & 909 & 11.83 & 1 & \\
\hline F08520-6850 W & ESO 60-IG016 & 0.046315 & 205.4 & 909 & 11.83 & 1 & \\
\hline $09022-3615$ & & 0.059641 & 267 & 1153 & 12.32 & 2 & \\
\hline F09437+0317 S & IC 563 & 0.020467 & 89 & 415 & 10.82 & $1(0)$ & $\mathrm{a}, \mathrm{c}, \mathrm{d}$ \\
\hline $\mathrm{F} 09437+0317 \mathrm{~N}$ & IC 564 & 0.020467 & 89 & 415 & 10.99 & $1(0)$ & $\mathrm{a}, \mathrm{c}, \mathrm{d}$ \\
\hline F10015-0614 & NGC 3110 & 0.016858 & 73.1 & 343 & 11.31 & 0 & \\
\hline F10038-3338 & ESO 374-IG032 & 0.034100 & 149.9 & 679 & 11.77 & 2 & \\
\hline F10257-4339 & NGC 3256 & 0.009354 & 40.4 & 192 & 11.69 & 2 & \\
\hline F10409-4556 & ESO 264-G036 & 0.021011 & 91.4 & 425 & 11.26 & 0 & \\
\hline F10567-4310 & ESO 264-G057 & 0.017199 & 74.6 & 350 & 11.07 & 0 & \\
\hline F11255-4120 & ESO 319-G022 & 0.016351 & 70.9 & 333 & 11.04 & 0 & \\
\hline F11506-3851 & ESO 320-G030 & 0.010781 & 46.6 & 221 & 11.30 & 0 & \\
\hline F12043-3140 S & ESO 440-IG058 & 0.023203 & 101.1 & 468 & 11.37 & 1 & $\mathrm{a}, \mathrm{d}$ \\
\hline F12043-3140 N & ESO 440-IG058 & 0.023203 & 101.1 & 468 & - & 1 & $\mathrm{a}, \mathrm{d}$ \\
\hline F12115-4656 & ESO 267-G030 & 0.018489 & 80.3 & 375 & 11.11 & 0 & \\
\hline $12116-5615$ & & 0.027102 & 118.5 & 545 & 11.61 & $2(0)$ & \\
\hline F12596-1529 & MCG 02-33-098 & 0.015921 & 69.0 & 324 & 11.07 & 1 & \\
\hline F13001-2339 & ESO 507-G070 & 0.021702 & 94.5 & 439 & 11.48 & $2(0 / 1)$ & \\
\hline F13229-2934 & NGC 5135 & 0.013693 & 59.3 & 280 & 11.29 & 0 & \\
\hline F14544-4255 E & IC 4518 & 0.015728 & 68.2 & 320 & 10.80 & 1 & $\mathrm{a}, \mathrm{d}$ \\
\hline F14544-4255 W & IC 4518 & 0.015728 & 68.2 & 320 & 10.80 & 1 & $\mathrm{a}, \mathrm{d}$ \\
\hline F17138-1017 & & 0.017335 & 75.2 & 352 & 11.41 & $2(0)$ & \\
\hline F18093-5744 S & IC 4689 & 0.017345 & 75.3 & 353 & - & 1 & $\mathrm{~b}, \mathrm{~d}$ \\
\hline F18093-5744 C & IC 4686 & 0.017345 & 75.3 & 353 & 10.87 & 1 & $\mathrm{~b}, \mathrm{~d}$ \\
\hline F18093-5744 N & IC 4687 & 0.017345 & 75.3 & 353 & 11.47 & 1 & $\mathrm{~b}, \mathrm{~d}$ \\
\hline F21130-4446 & & 0.092554 & 423.9 & 1722 & 12.09 & 2 & \\
\hline F21453-3511 & NGC 7130 & 0.016151 & 70.0 & 329 & 11.41 & 2 & \\
\hline F22132-3705 & IC 5179 & 0.011415 & 49.3 & 234 & 11.22 & 0 & \\
\hline F22491-1808 & & 0.077760 & 352.5 & 1471 & 12.17 & 1 & \\
\hline F23128-5919 & AM 2312-591 & 0.044601 & 197.5 & 878 & 12.06 & 1 & \\
\hline
\end{tabular}

Notes. Column (1): object designation in the Infrared Astronomical Satellite (IRAS) Faint Source Catalog (FSC). Column (2): other identification. Column (3): redshift from the NASA Extragalactic Database (NED). Column (4): luminosity distance assuming a $\Lambda$ DCM cosmology with $H_{0}=$ $70 \mathrm{~km} \mathrm{~s}^{-1} \mathrm{Mpc}^{-1}, \Omega_{\mathrm{M}}=0.3$, and $\Omega_{\Lambda}=0.7$, using the E. L. Wright Cosmology calculator, which is based on the prescription given by Wright (2006). Column (5): scale. Column (6): infrared luminosity $\left(L_{\mathrm{IR}}=L(8-1000) \mu \mathrm{m}\right)$ in units of solar bolometric luminosity, calculated using the fluxes in the four IRAS bands as given in Sanders et al. (2003) when available. Otherwise, the standard prescription given in Sanders \& Mirabel (1996) with the values in the IRAS Point and Faint Source catalogs was used. Column (7): morphological class defined as follows: 0 identifies isolated objects, 1 pre-coalescence systems, and 2 stands for merger objects. For those objects for which the morphological classification is uncertain, the various possible classes are shown in the table with the preferred morphological classification indicated in the first place and alternative classification within brackets (see text for further details). Column (8): notes with the following code: (a) system composed of two galaxies. (b) system composed of three galaxies. (c) there are two VIMOS pointings for the northern source. (d) interacting system (i.e., see notes $a$ and $b$ ) for which the total infrared luminosity $L_{\mathrm{IR}}$ could be approximately assigned among the members of the system according to the Spitzer/MIPS photometry. For further details, see B13. 
A\&A 591, A85 (2016)

Table A.2. Comparison of the different (morphological and kinematic) classifications of the local (U)LIRG sample.

\begin{tabular}{|c|c|c|c|c|}
\hline $\begin{array}{l}\text { ID1 } \\
\text { IRAS } \\
\text { (1) }\end{array}$ & $\begin{array}{c}\text { Morphological } \\
\text { classification } \\
(2)\end{array}$ & $\begin{array}{l}\text { Visual kinematic } \\
\text { classification } \\
\text { (3) }\end{array}$ & $\begin{array}{c}\text { Kinemetry } \\
\text { value of } K_{\text {tot }} \\
\text { (4) }\end{array}$ & $\begin{array}{l}\text { Kinemetry } \\
\text { classification } \\
\text { (5) }\end{array}$ \\
\hline F01159-4443 S & 1 & PD & 0.25 & Disk $^{\star}$ \\
\hline F01159-4443 N & 1 & PD & 0.12 & Disk \\
\hline F01341-3735 S & 1 & PD & 0.14 & Disk \\
\hline F01341-3735 N & 1 & $\mathrm{PD}$ & 0.06 & Disk \\
\hline F04315-0840 & 2 & $\mathrm{CK}$ & 0.2 & $\operatorname{Disk}^{\star}$ \\
\hline F05189-2524 & 2 & $\mathrm{CK}$ & 1.14 & Merger \\
\hline F06035-7102 & 1 (ongoing) & $\mathrm{CK}$ & 2.91 & Merger \\
\hline F06076-2139 S & 1 & PD & 0.1 & Disk \\
\hline F06076-2139 N & 1 & PD & 0.09 & Disk \\
\hline F06206-6315 & 1 (ongoing) & $\mathrm{PD}$ & 1.26 & Merger \\
\hline F06259-4780 S & 1 & $\mathrm{RD}$ & 0.09 & Disk \\
\hline F06259-4780 C & 1 & $\mathrm{RD}$ & 0.10 & Disk \\
\hline F06259-4780 N & 1 & $\mathrm{RD}$ & 0.13 & Disk \\
\hline F06295-1735 & 0 & $\mathrm{PD}$ & 0.20 & Disk $^{\star}$ \\
\hline F06592-6313 & 0 & $\mathrm{PD}$ & 0.13 & Disk \\
\hline F07027-6011 S & 0 & $\mathrm{RD}$ & 0.14 & Disk \\
\hline F07027-6011 N & 0 & $\mathrm{PD}$ & 0.06 & Disk \\
\hline F07160-6215 & 0 & $\mathrm{PD}(\mathrm{CK})$ & 0.47 & Disk $^{\star}$ \\
\hline 08355-4944 & 2 & $\mathrm{PD}$ & 0.27 & Disk $^{\star}$ \\
\hline 08424-3130 S & 1 & $\mathrm{PD}$ & 0.17 & Disk \\
\hline $08424-3130 \mathrm{~N}$ & 1 & - & - & - \\
\hline F08520-6850 E & 1 & $\mathrm{RD}$ & 0.45 & Disk $^{\star}$ \\
\hline F08520-6850 W & 1 & $\mathrm{PD}(\mathrm{RD})$ & 0.07 & Disk \\
\hline 09022-3615 & 2 & $\mathrm{CK}$ & 1.04 & Merger \\
\hline F09437+0317 S & $1(0)$ & $\mathrm{RD}$ & 0.08 & Disk \\
\hline $\mathrm{F} 09437+0317 \mathrm{~N}$ & $1(0)$ & $\mathrm{RD}$ & 0.06 & Disk \\
\hline F10015-0614 & 0 & $\mathrm{PD}$ & 0.10 & Disk \\
\hline F10038-3338 & 2 & $\mathrm{CK}$ & 0.35 & Disk $^{\star}$ \\
\hline F10257-4339 & 2 & $\mathrm{PD}$ & 5.88 & Merger \\
\hline F10409-4556 & 0 & $\mathrm{RD}$ & 0.16 & Disk \\
\hline F10567-4310 & 0 & $\mathrm{RD}$ & 0.05 & Disk \\
\hline F11255-4120 & 0 & $\mathrm{PD}$ & 0.07 & Disk \\
\hline F11506-3851 & 0 & $\mathrm{RD}$ & 0.08 & Disk \\
\hline F12043-3140 S & 1 & $\mathrm{PD}$ & 0.13 & Disk \\
\hline F12043-3140 N & 1 & $\mathrm{PD}(\mathrm{CK})$ & 0.71 & Merger ${ }^{\star}$ \\
\hline F12115-4656 & 0 & $\mathrm{RD}$ & 0.02 & Disk \\
\hline $12116-5615$ & $2(0)$ & PD & 0.28 & $\operatorname{Disk}^{\star}$ \\
\hline F12596-1529 & 1 (ongoing) & - & 3.01 & Merger \\
\hline F13001-2339 & $2(0 / 1)$ & $\mathrm{CK}$ & 0.93 & Merger $^{\star}$ \\
\hline F13229-2934 & 0 & $\mathrm{CK}$ & 0.25 & $\operatorname{Disk}^{\star}$ \\
\hline F14544-4255 E & 1 & $\mathrm{PD}$ & 0.15 & Disk \\
\hline F14544-4255 W & 1 & $\mathrm{CK}(\mathrm{PD})$ & 0.23 & Disk $^{\star}$ \\
\hline F17138-1017 & $2(0)$ & PD & 0.22 & Disk $^{\star}$ \\
\hline F18093-5744 S & 1 & $\mathrm{RD}$ & 0.06 & Disk \\
\hline F18093-5744 C & 1 & $\mathrm{CK}(\mathrm{PD})$ & 0.19 & Disk $^{\star}$ \\
\hline F18093-5744 N & 1 & $\mathrm{RD}$ & 0.05 & Disk \\
\hline F21130-4446 & 2 & $\mathrm{CK}$ & 0.34 & Disk $^{\star}$ \\
\hline F21453-3511 & 2 & $\mathrm{PD}$ & 0.20 & Disk $^{\star}$ \\
\hline F22132-3705 & 0 & $\mathrm{RD}$ & 0.04 & Disk \\
\hline F22491-1808 & 1 (ongoing) & $\mathrm{CK}(\mathrm{PD})$ & 0.95 & Merger \\
\hline F23128-5919 & 1 (ongoing) & $\mathrm{CK}$ & 3.62 & Merger \\
\hline
\end{tabular}

Notes. Column (1): object designation in the Infrared Astronomical Satellite (IRAS) Faint Source Catalog (FSC). Column (2): morphological class as defined in previous works (i.e., A08, MI10, RZ11, and B13) as follows: 0 identifies isolated object, 1 pre-coalescence system, and 2 stands for post-coalescence merger. Column (3): visual kinematic classification as in B13. RD stands for rotating disk, PD perturbed disk, and CK are systems with complex kinematics. Column (4): Kinemetry value of the total kinematic asymmetry $K_{\text {tot }}$ defined in Sect. 3.4 for the observed systems as derived according to the "weighted" method. Column (5): Kinemetry classification of the observed systems according to the "weighted" method $\left(K_{\mathrm{F}}=0.19\right)$. Galaxies classified as disk ${ }^{\star}$ or merger ${ }^{\star}$ are those lying in the transition region (see Sect. 3.4). No kinemetry analysis was performed for the galaxy IRAS $08424-3130 \mathrm{~N}$ since it is located in the edge of the VIMOS FoV. 